This item was submitted to Loughborough's Research Repository by the author.

Items in Figshare are protected by copyright, with all rights reserved, unless otherwise indicated.

\title{
Adaptive nonlinear relative motion control of quadrotors in autonomous shipboard landings
}

PLEASE CITE THE PUBLISHED VERSION

https://doi.org/10.1016/j.jfranklin.2020.10.014

PUBLISHER

Elsevier

VERSION

AM (Accepted Manuscript)

\section{PUBLISHER STATEMENT}

This paper was accepted for publication in the journal Journal of the Franklin Institute and the definitive published version is available at https://doi.org/10.1016/j.jfranklin.2020.10.014

LICENCE

CC BY-NC-ND 4.0

\section{REPOSITORY RECORD}

Sun, Liang, Yanting Huang, Zewei Zheng, Bing Zhu, and Jingjing Jiang. 2020. "Adaptive Nonlinear Relative Motion Control of Quadrotors in Autonomous Shipboard Landings”. Loughborough University. https://hdl.handle.net/2134/13106849.v1. 


\title{
Adaptive Nonlinear Relative Motion Control of Quadrotors in Autonomous Shipboard Landings
}

\author{
Liang Sun ${ }^{\mathrm{a}, \mathrm{b}, *}$, Yanting Huang ${ }^{\mathrm{c}}$, Zewei Zheng ${ }^{\mathrm{d}}$, Bing Zhu ${ }^{\mathrm{d}}$, Jingjing Jiang ${ }^{\mathrm{e}}$ \\ ${ }^{a}$ Key Laboratory of Knowledge Automation for Industrial Processes of Ministry of Education, \\ School of Automation and Electrical Engineering, University of Science and Technology Beijing, Beijing 100083, China \\ ${ }^{b}$ Institute of Artificial Intelligence, University of Science and Technology Beijing, Beijing 100083, China \\ ${ }^{c}$ School of Aeronautical Science and Engineering, Beihang University, Beijing 100191, China \\ ${ }^{d}$ The Seventh Research Division, School of Automation Science and Electrical Engineering, Beihang University, Beijing 100191, P.R.China \\ ${ }^{e}$ Department of Aeronautical and Automotive Engineering, Loughborough University, Leicestershire, LE11 3TU, United Kingdom
}

\begin{abstract}
The relative motion control for the unmanned quadrotors autonomous shipboard landings is investigated in this study. The shipboard landing missions are divided into two phases including the position approaching phase and the vertical landing phase. Relative pose kinematics and dynamics are modeled in the quadrotor's body-fixed frame, where the kinematic couplings resulted from the difference between ship's centre of mass and desired landing site and the unknown external disturbances of two vehicles are considered in the modeling and control design. In the position approaching phase, because of the under-actuated property of quadrotors, the adaptive backstepping technique is combined with an auxiliary system and a command filter to develop the guidance and control laws. Subsequently, the relative altitude-attitude controller is designed for the vertical landing phase. Stability analysis shows that the position approaching errors in the first phase and the landing errors in the second phase ultimately converge to small neighborhoods of zero, and numerical simulation validates the effectiveness of the proposed strategy.
\end{abstract}

Keywords: Quadrotor control, autonomous shipboard landings, relative motion control, nonlinear systems control, disturbances

\section{Introduction}

In recent decades, many studies have been performed on the design, analysis, and operation of autonomous unmanned helicopters $[1,2]$. In fact, quad-rotor helicopters with strong abilities of maneuvers and tracking are very suitable for maritime missions [3, 4], such as for autonomous shipboard landing operations [5]. However, shipboard landing is one of the most challenging missions for the helicopters due to the natural complexity involving ships and quadrotors in relative motion, wind blowing from any direction, and strong ocean current interaction on the ship [6]. Quadrotors can achieve low-dynamic tracking missions and operate in a ship deck, but the ship deck is always shaking due to the rough disturbances generated from the uncertain wind and ocean current. Furthermore, the controller design for autonomous shipboard landings is very challenging since relative pose motion model between two vehicles is highly nonlinear and coupled. In particular, the active quadrotor is a typical under-actuated system because of smaller number of control inputs than the number of generalized coordinates [7]. Moreover, the desired landing site of the quadrotor is generally not located at the centre of the mass of the ship. Then, the relative pose dynamics between landing site and the quadrotor and the model-based controller design for the shipboard landing mission are very complicated. Therefore, it is very necessary to

\footnotetext{
${ }^{*}$ Corresponding author.

Email address: liangsun@ustb.edu.cn (Liang Sun )
}

study the advanced relative pose controller design for the autonomous high-precision shipboard landing operations.

As a hot topic in control engineering, a number of studies on autonomous shipboard helicopter landing have been studied in recent years. The robust controller for helicopters was studied in [8]. This controller takes into consideration unexpected disturbances and turbulence over the sea. To achieve a practical model and system, a mathematical model of the ondeck helicopter/ship dynamic interface was developed by [9]. Then, the study in [7] allowed precise controlled landing in difficult situations by connecting the moving shipboard and the aircraft with a physical tether. Based on the image features as references while filtering its movements to get a smooth helicopter trajectory compared to the targeted ship, a method to set two controllers based on a translational rate command law and a common advanced speed control law for modern helicopters were presented by [10]. Furthermore, to improve the landing precision of the helicopter, a vision-based auto landing system was proposed in [11] for unmanned helicopter on a ship. Based on fuzzy recognition, Kalman filter and proportional integral controller in [11], the unmanned helicopter can track the moving target and land on it. Moreover, a monocular vision system was developed in [12] for an unmanned aerial vehicle landing on a ship's deck, and the four-degrees-of-freedom pose of the vehicle with respect to the ship was estimated. Besides, the guidance law of the helicopter is also important for the high-precision landing missions, then visual/inertial guidance 
strategy [13] and vision/radar/INS integrated guidance strategy [14] were developed respectively to improve the hardware performance of autonomous shipboard landings. The two-phases planning method for the carrier landing missions was developed in [15], where the pigeon inspired optimization algorithm was used to reduce the terminal position errors and adapt to the different environment. The autonomous landing operation proceeds in three phases: the search phase, the homing phase, and the landing phase [16], where a downward-looking single camera was used for determining helicopter's position relative to the moving platform. A method to assessment of mission success for helicopter landing on a ship was developed in [17].

The aforementioned studies mainly focus on the navigation and planning system designs for the helicopter shipboard landings. Various studies on robust landing controller design to suppress the model uncertainty of the helicopter and the disturbances involved in over sea operations were also reported recently. The application of the neural networks and fuzzy logicbased robust intelligent controller on the automated landing of unmanned helicopters was studied by [18] to compensate the model uncertainties. Then, the study in [19] focused on the uncertainty compensation of the autonomous shipboard landing control strategy, where a disturbance estimator was develope$\mathrm{d}$ to design the robust backstepping controller. Meanwhile, a control architecture that consists of a motion estimation module, a trajectory generation module and a tracking control module was proposed in [20] to achieve the high-performance auto landing missions. In particular, the time-optimal trajectory tracking controller was developed for quadrotors landing on a moving platform. A dynamic image-based visual servo control strategy was proposed for a helicopter landing on a moving vehicle by [21], so that the control performance is improved clearly based on the visual measurement. A disturbance attenuation controller for horizontal position stabilization was developed in [22] for hover and automatic landings of a helicopter approaching to the landing deck. An invariant ellipsoid-based method for the controller design and gain synthesis of a lowcost quadrotor autonomous landing system subjected to wind disturbance and measurement noise was proposed by [23]-[25]. Then, a class of relative motion model was developed for the shipboard landing missions by [26]-[28], and the adaptive nonlinear controllers were presented to ensure the success of the helicopter landing on the deck in rough sea. However, the dynamic couplings between the relative position and relative attitude motions were ignored, while the under-actuated property of the aeronautical vehicles was also insufficiently considered in the previous works.

This study mainly focuses on the relative pose control problem between the quadrotor and ship for the shipboard landing missions. Enlightened by the previous studies in [26] and [27], the shipboard landing mission is also divided into two phases including the autonomous position approaching phase and the autonomous vertical landing phase in this study. The main contributions of this work are stated as follows.

- The novel relative pose kinematics and dynamics are modeled in the quadrotor's body-fixed frame, where the atti- tude motion of two vehicles are described by the modified Rodrigues parameter because of its non-singularity and non-redundant properties. In particular, the landing site on the deck is considered into the modelling of relative position dynamics to describe the relative motion precisely, and the bounded external disturbances generated from the changing environment are also considered in the relative motion of two vehicles.

- Based on the different state information, two controller$\mathrm{s}$ are developed respectively for the position approaching phase and the vertical landing phase in view of the underactuated property of the quadrotor. Specifically, in the position approaching phase, the control singularity of the quadrotor is avoided by using the saturated force inputs and the bounded auxiliary system, while the second-order command filter is also employed to avoid the complicated time-derivative calculations in the relative motion controller design.

- It is proved in the Lyapunov framework that the position tracking errors in autonomous position approaching phase and the terminal landing altitude-attitude errors in autonomous vertical landing phase uniformly ultimately converge to the small neighborhoods of zero by tuning suitable designing parameters. Simulation results validate the effectiveness of the proposed approach.

This paper is organized as follows. Section 2 presents the mathematical modeling and control mission for autonomous quadrotor shipboard landing operations. Section 3 provides explicit controller design procedures, and the stability result of the closed-loop system is also rigorously analyzed. Numerical simulations are performed in Section 4 to verify the theoretic developments. Finally, conclusions are shown in Section 5.

Throughout the paper, the skew-symmetric matrix $S(\boldsymbol{a}) \in$ $\mathbb{R}^{3 \times 3}$ derived from any vector $\boldsymbol{a}=\left[a_{1}, a_{2}, a_{3}\right]^{\mathrm{T}} \in \mathbb{R}^{3}$ is defined by $S(\boldsymbol{a})=\left[0,-a_{3}, a_{2} ; a_{3}, 0,-a_{1} ;-a_{2}, a_{1}, 0\right]$. It satisfies $\boldsymbol{a}^{\mathrm{T}} S(\boldsymbol{a})=0,\|S(\boldsymbol{a})\|=\|\boldsymbol{a}\|$, and $S(\boldsymbol{a}) \boldsymbol{b}=-S(\boldsymbol{b}) \boldsymbol{a}$, $\boldsymbol{b}^{\mathrm{T}} S(\boldsymbol{a}) \boldsymbol{b}=0$ for any $\boldsymbol{b} \in \mathbb{R}^{3}$. $\|\boldsymbol{a}\|$ denotes vector 2-norm of $\boldsymbol{a},\|A\|$ represents the induced matrix 2-norm of $A$. $I_{n}$ and $O_{n}$ are $n \times n$ unit and zero matrices, respectively. Moreover, $\tanh (c)=\left[\tanh \left(c_{1}\right), \tanh \left(c_{2}\right), \cdots, \tanh \left(c_{n}\right)\right]^{\mathrm{T}}$ for any vector $c=\left[c_{1}, c_{2}, \cdots, c_{n}\right]^{\mathrm{T}}$.

\section{Problem Description}

We investigate the control problem of an autonomous quadrotor landing on the deck of a ship in the rough sea. Due to the complicated relative motion model between the underactuated quadrotor and the uncontrolled ship, the quadrotor's landing mission in this study is divided into two coherent phases, such as the autonomous position approaching phase shown in Figure 1 and the autonomous vertical landing phase shown in Figure 2. In Figures 1 and 2, $\mathcal{F}_{i} \triangleq\left\{\mathbf{O} x_{i} y_{i} z_{i}\right\}$ is the Earth reference frame with its origin $\mathbf{O}$ locating at a fixed point. The $z_{i}$ axis points upright vertically, $x_{i}$ points to the north, and $y_{i}$ 
completes the triad by right-hand rule. $\mathcal{F}_{h} \triangleq\{\mathbf{H} x y z\}$ is quadrotor's body-fixed frame, where origin $\mathbf{H}$ locates at the centre of the mass of fuselage, $x$ points to the fuselage head, $z$ axis is perpendicular to $x$ axis and points upright, and $y$ axis is determined by right-hand rule. $\mathcal{F}_{s} \triangleq\left\{\mathbf{S} x_{t} y_{t} z_{t}\right\}$ is ship's body-fixed frame, where origin $\mathbf{S}$ is the centre of mass of ship, $x_{s}$ axis points to the front of the ship, $z_{s}$ axis is perpendicular to $x_{s}$ axis and points upright, and $y_{s}$ axis completes the triad by right-hand rule. $\mathbf{P}$ and $\mathbf{P}^{\prime}$ are fixed points with respect to $\mathbf{S}$, where point $\mathbf{P}$ is the desired position of the quadrotor with only small height with respect to the final landing site $\mathbf{P}^{\prime}$ on the deck; $\left\{\boldsymbol{r}, \boldsymbol{r}_{e}, \boldsymbol{r}_{e}^{\prime}\right\}$ and $\left\{\boldsymbol{r}_{s}, \boldsymbol{r}_{p_{s}}, \boldsymbol{p}_{s}, \boldsymbol{r}_{p_{s}}^{\prime}, \boldsymbol{p}_{s}^{\prime}\right\}$ are related position vectors expressed in frames $\mathcal{F}_{h}$ and $\mathcal{F}_{s}$, respectively. Specifically, as shown in Figure 1 , the objective of the controller design in autonomous position approaching phase is to control the quadrotor so that its centre of mass $\mathbf{H}$ tracks point $\mathbf{P}$ to get ready for the consequently vertical landing phase. Then, as shown in Figure 2, after the quadrotor's centre of mass $\mathbf{H}$ arriving at point $\mathbf{P}$, namely $\mathbf{H}$ coinciding with $\mathbf{P}$, the control objective of the autonomous vertical landing phase is to control the quadrotor's height so that relative altitude between the centre of mass $\mathbf{H}$ and point $\mathbf{P}^{\prime}$ is zero, while the frame $\mathcal{F}_{h}$ tracks the frame $\mathcal{F}_{s}$.

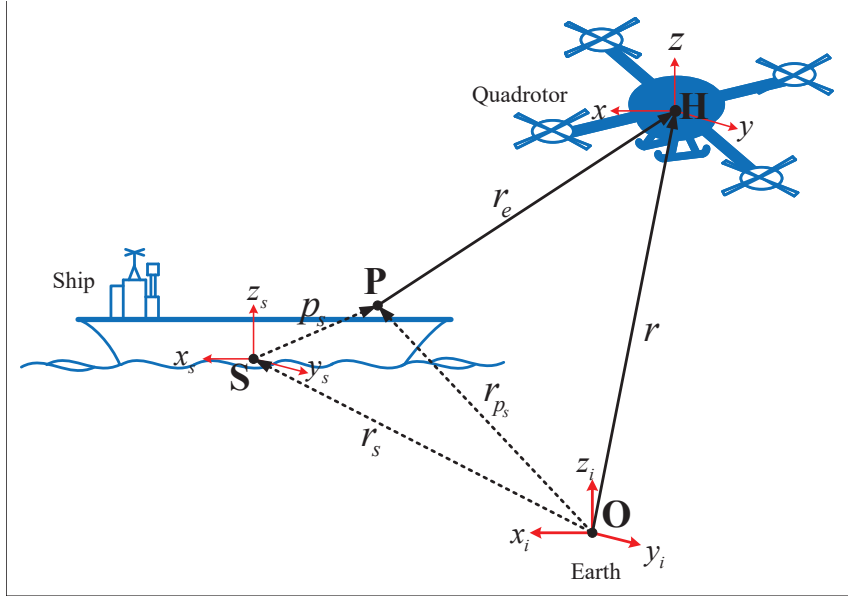

Figure 1: Autonomous position approaching phase in shipboard landings.

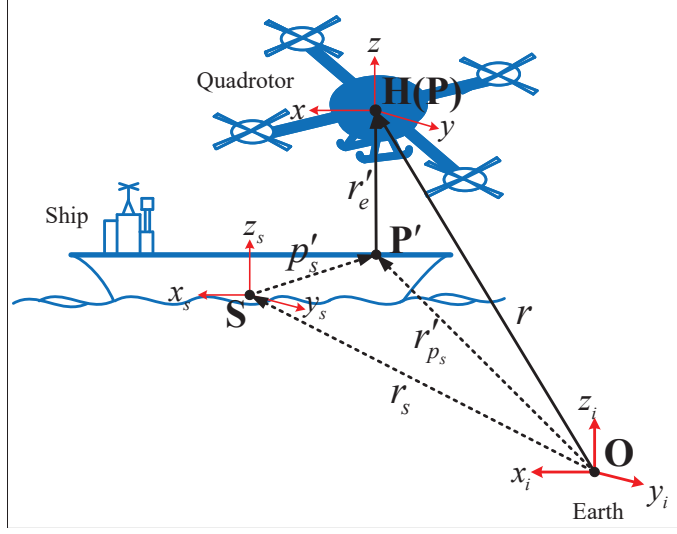

Figure 2: Autonomous vertical landing phase in shipboard landings.
Remark 1. Since the autonomous landing missions are generally performed when the quadrotor is far from the ship deck, so the trajectory tracking should be firstly achieved, then the quadrotor could perform the final landing operations when the quadrotor is very near the ship deck. The most important reason for dividing the landing missions into two phases is the under-actuated control property of the autonomous quadrotor. Since the position tracking phase should ensure that the quadrotor completely track the three-dimensional position of the ship deck, so that the attitude trajectory of the quadrotor cannot be synchronized with the ship in real time. Furthermore, after completing the tracjecotry tracking phase, the quadrotor is very near the final landing site of the ship in the second phase, so that the landing operations mainly focus on the relative altitude regulation and attitude synchronization of two vehicles in a short time. Thus the quadrotor should switch its autonomous control modes from the trajectory tracking to the altitude-attitude synchronization, so that the autonomous landing mission is divided into two successive phases to facilitate control system design of the quadrotor.

\subsection{Nonlinear Dynamics of Quadrotors and Ships}

The position motion of centre of mass $\mathbf{H}$ and the attitude motion of $\mathcal{F}_{h}$ with respect to $\mathcal{F}_{i}$ for a quadrotor are described by following kinematics and dynamics expressed in frame $\mathcal{F}_{h}$, if the modified Rodrigues parameters(MRP) are used for attitude parametrization [29].

$$
\left\{\begin{array}{l}
\dot{\boldsymbol{r}}=\boldsymbol{v}-S(\boldsymbol{\omega}) \boldsymbol{r} \\
m \dot{\boldsymbol{v}}+m S(\omega) \boldsymbol{v}+m g R^{\mathrm{T}} \boldsymbol{e}_{3}=T \boldsymbol{e}_{3}+\boldsymbol{d}_{h} \\
\dot{\boldsymbol{\sigma}}=G(\boldsymbol{\sigma}) \boldsymbol{\omega} \\
J \dot{\omega}+S(\boldsymbol{\omega}) J \boldsymbol{\omega}=\boldsymbol{\tau}+\boldsymbol{w}_{h}
\end{array}\right.
$$

where $G(\sigma)=\frac{1}{4}\left[\left(1-\sigma^{\mathrm{T}} \boldsymbol{\sigma}\right) I_{3}+2 S(\sigma)+2 \sigma \sigma^{\mathrm{T}}\right], g$ is gravity acceleration, $\boldsymbol{e}_{3}=[0,0,1]^{\mathrm{T}} ; \boldsymbol{r} \in \mathbb{R}^{3}$ is the position and $\boldsymbol{\sigma}$ is the MRP attitude; $\boldsymbol{v}, \omega \in \mathbb{R}^{3}$ are linear and angular velocities, respectively; $T \in \mathbb{R}$ is the control thrust, and $\tau \in \mathbb{R}^{3}$ is the three-axes torque vector; $m \in \mathbb{R}$ and $J \in \mathbb{R}^{3 \times 3}$ are the mass and the positive definite symmetric inertia matrix of the helicopter, respectively; $R$ is the rotation matrix from $\mathcal{F}_{h}$ to $\mathcal{F}_{i} ; \boldsymbol{d}_{h}, \boldsymbol{w}_{h} \in \mathbb{R}^{3}$ are the unmodeled disturbances.

The kinematics and dynamics of an uncontrolled ship in rough sea can be simply described in frame $\mathcal{F}_{s}$ as $[27,30]$

$$
\left\{\begin{array}{l}
\dot{\boldsymbol{r}}_{s}=\boldsymbol{v}_{s}-S\left(\boldsymbol{\omega}_{s}\right) \boldsymbol{r}_{s} \\
m_{s} \dot{\boldsymbol{v}}_{s}+m_{s} S\left(\omega_{s}\right) \boldsymbol{v}_{s}=\boldsymbol{d}_{s} \\
\dot{\boldsymbol{\sigma}}_{s}=G\left(\boldsymbol{\sigma}_{s}\right) \boldsymbol{\omega}_{s} \\
J_{s} \dot{\boldsymbol{\omega}}_{s}+S\left(\boldsymbol{\omega}_{s}\right) J_{s} \boldsymbol{\omega}_{s}=\boldsymbol{w}_{s}
\end{array}\right.
$$

where $G\left(\sigma_{s}\right)=\frac{1}{4}\left[\left(1-\sigma_{s}^{\mathrm{T}} \boldsymbol{\sigma}_{s}\right) I_{3}+2 S\left(\boldsymbol{\sigma}_{s}\right)+2 \sigma_{s} \boldsymbol{\sigma}_{s}^{\mathrm{T}}\right], \boldsymbol{r}_{s} \in \mathbb{R}^{3}$ and $\sigma_{s}$ are position and attitude of the ship, respectively; $\boldsymbol{v}_{s}, \omega_{s} \in$ $\mathbb{R}^{3}$ are linear and angular velocities of the ship, respectively; $\boldsymbol{d}_{s}, \boldsymbol{w}_{s} \in \mathbb{R}^{3}$ are the external force and torque, respectively; $m_{s} \in$ $\mathbb{R}$ and $J_{s} \in \mathbb{R}^{3 \times 3}$ are mass and inertial matrix of the target, respectively.

Remark 2. The unmodeled disturbances $\boldsymbol{d}_{h}$ and $\boldsymbol{w}_{h}$ in (1) are generated from the aerodynamic effect of the main rotor and 
the fuselage. The external force $\boldsymbol{d}_{s}$ and external torque $\boldsymbol{w}_{s}$ in (2) mainly include the influence of gravity, buoyancy, atmospheric drag, hydrodynamic damping effect, added mass and inertia forces. The ship's six-degrees-of-freedom motion is modeled as (2) with simplified form, since this study mainly focus on the unmanned quadrotor's control system design, and this ship's model (2) is almost enough to reflect the motion feature of the ship in the autonomous landing missions. In fact, the explicitly six-degrees-of-freedom motion model of the ship can be found in [30]. Moreover, according to the statement in [29], the shadow MRP set can be exploited to yield a globally non-singular attitude description with a minimal three-parameter coordinate set at the expense of a discontinuity. To avoid the singularity, the MRP set is switched to the shadow set before reaching the singularity. A convenient switching condition is the unit magnitude surface $\|\sigma\|=1$, such that the composite MRP description always satisfies $\|\sigma\| \leq 1$.

\subsection{Relative Rotational and Translational Dynamics for Au- tonomous Shipboard Landings}

Let the MRP relative attitude $\sigma_{e}$ be [29]

$$
\sigma_{e}=\frac{\sigma_{s}\left(\sigma^{\mathrm{T}} \sigma-1\right)+\sigma\left(1-\sigma_{s}^{\mathrm{T}} \sigma_{s}\right)-2 S\left(\sigma_{s}\right) \sigma}{1+\sigma_{s}^{\mathrm{T}} \sigma_{s} \sigma^{\mathrm{T}} \sigma+2 \sigma_{s}^{\mathrm{T}} \sigma}
$$

and the rotation matrix from $\mathcal{F}_{s}$ to $\mathcal{F}_{h}$ is defined by

$$
R_{e}=I_{3}-\frac{4\left(1-\sigma_{e}^{\mathrm{T}} \sigma_{e}\right)}{\left(1+\sigma_{e}^{\mathrm{T}} \sigma_{e}\right)^{2}} S\left(\sigma_{e}\right)+\frac{8 S^{2}\left(\sigma_{e}\right)}{\left(1+\sigma_{e}^{\mathrm{T}} \sigma_{e}\right)^{2}}
$$

According to Figure 1, the position and velocity of point $\mathbf{P}$ represented in frame $\mathcal{F}_{s}$ are given by

$$
\boldsymbol{r}_{p_{s}}=\boldsymbol{r}_{s}+\boldsymbol{p}_{s}, \quad \boldsymbol{v}_{p_{s}}=\boldsymbol{v}_{s}+S\left(\omega_{s}\right) \boldsymbol{p}_{s}
$$

where $\boldsymbol{p}_{s} \in \mathbb{R}^{3}$ is a constant vector in frame $\mathcal{F}_{s}$. The relative position, relative velocity, and relative angular velocity are defined in frame $\mathcal{F}_{h}$ as

$$
\omega_{e}=\omega-R_{e} \omega_{s}, \boldsymbol{r}_{e}=\boldsymbol{r}-R_{e} \boldsymbol{r}_{p_{s}}, \boldsymbol{v}_{e}=\boldsymbol{v}-R_{e} \boldsymbol{v}_{p_{s}}
$$

Substituting (6) into (1) and using identities $\dot{R}_{e}=-S\left(\omega_{e}\right) R_{e}$, $\dot{\boldsymbol{r}}_{p_{s}}=\boldsymbol{v}_{p_{s}}-S\left(\omega_{s}\right) \boldsymbol{r}_{p_{s}}$ and $R_{e}^{-1}=R_{e}^{\mathrm{T}}$ yield the relative motion equations in frame $\mathcal{F}_{h}$ as

$$
\left\{\begin{aligned}
\dot{\boldsymbol{r}}_{e} & =\boldsymbol{v}_{e}-S(\boldsymbol{\omega}) \boldsymbol{r}_{e} \\
\dot{\boldsymbol{v}}_{e} & =-\left[S(\omega) \boldsymbol{v}+g R^{\mathrm{T}} \boldsymbol{e}_{3}+R_{e} \dot{\boldsymbol{v}}_{p_{s}}-S\left(\boldsymbol{\omega}_{e}\right)\left(\boldsymbol{v}-\boldsymbol{v}_{e}\right)\right]+\frac{T}{m} \boldsymbol{e}_{3}+\frac{1}{m} \boldsymbol{d}_{h} \\
\dot{\boldsymbol{\sigma}}_{e} & =G\left(\boldsymbol{\sigma}_{e}\right) \boldsymbol{\omega}_{e} \\
J \dot{\boldsymbol{\omega}}_{e} & =-S(\omega) J \omega-J\left[R_{e} \dot{\omega}_{s}+S(\omega) \omega_{e}\right]+\tau+\boldsymbol{w}_{h}
\end{aligned}\right.
$$

where kinematic Jacobian matrix $G\left(\sigma_{e}\right)=\frac{1}{4}\left[\left(1-\sigma_{e}^{\mathrm{T}} \sigma_{e}\right) I_{3}+\right.$ $2 S\left(\sigma_{e}\right)+2 \sigma_{e} \sigma_{e}^{\mathrm{T}}$ ] is nonsingular [29]; $R_{e} \dot{\boldsymbol{v}}_{p_{s}}$ is calculated from (5), (2), (6) and $R_{e} S(\boldsymbol{a})=S\left(R_{e} \boldsymbol{a}\right) R_{e}$ for any $\boldsymbol{a} \in \mathbb{R}^{3}$ as $R_{e} \dot{\boldsymbol{v}}_{p_{s}}=R_{e}\left[\dot{\boldsymbol{v}}_{s}+S\left(\dot{\boldsymbol{\omega}}_{s}\right) \boldsymbol{p}_{s}\right]=-R_{e} S\left(\omega_{s}\right) \boldsymbol{v}_{s}+\frac{1}{m_{s}} R_{e} \boldsymbol{d}_{s}-R_{e} S\left(\boldsymbol{p}_{s}\right) \dot{\boldsymbol{\omega}}_{s}=$ $-S\left(R_{e} \omega_{s}\right)\left[R_{e} v_{p_{s}}-R_{e} S\left(\omega_{s}\right) p_{s}\right]+\frac{1}{m_{s}} R_{e} d_{s}-R_{e} S\left(p_{s}\right) \dot{\omega}_{s}=-S(\omega-$
$\left.\omega_{e}\right)\left[v-v_{e}-S\left(\omega-\omega_{e}\right) R_{e} p_{s}\right]+\frac{1}{m_{s}} R_{e} d_{s}-R_{e} S\left(p_{s}\right) \dot{\omega}_{s}$, and $\dot{\omega}_{s}$ is calculated by

$$
\begin{aligned}
\dot{\omega}_{s} & =-J_{s}^{-1} S\left(\omega_{s}\right) J_{s} \omega_{s}+J_{s}^{-1} \boldsymbol{w}_{s} \\
& =-J_{s}^{-1} S\left(R_{e}^{\mathrm{T}}\left(\omega-\omega_{e}\right)\right) J_{s} R_{e}^{\mathrm{T}}\left(\omega-\omega_{e}\right)+J_{s}^{-1} \boldsymbol{w}_{s}
\end{aligned}
$$

Thus, equation (7) can be rewritten as

$$
\begin{gathered}
\dot{\boldsymbol{r}}_{e}=\boldsymbol{v}_{e}-S(\boldsymbol{\omega}) \boldsymbol{r}_{e} \\
\dot{\boldsymbol{v}}_{e}=-\boldsymbol{h}-g R^{\mathrm{T}} \boldsymbol{e}_{3}+\boldsymbol{d}_{1}+\boldsymbol{n}_{1}+\frac{T}{m} \boldsymbol{e}_{3} \\
\dot{\boldsymbol{\sigma}}_{e}=G\left(\boldsymbol{\sigma}_{e}\right) \boldsymbol{\omega}_{e} \\
J \dot{\boldsymbol{\omega}}_{e}=-S(\boldsymbol{\omega}) J \boldsymbol{\omega}-J S(\boldsymbol{\omega}) \boldsymbol{\omega}_{e}+\boldsymbol{n}_{2}+\boldsymbol{d}_{2}+\boldsymbol{\tau}
\end{gathered}
$$

where $\boldsymbol{h}=S(\boldsymbol{\omega}) \boldsymbol{v}_{e}+S^{2}\left(\boldsymbol{\omega}-\omega_{e}\right) R_{e} \boldsymbol{p}_{s}, \boldsymbol{d}_{1}=\frac{\boldsymbol{d}_{h}}{m}-\frac{R_{e} \boldsymbol{d}_{s}}{m_{s}}+$ $R_{e} S\left(\boldsymbol{p}_{s}\right) J_{s}^{-1} \boldsymbol{w}_{s}, \boldsymbol{d}_{2}=\boldsymbol{w}_{h}-R_{e} J_{s}^{-1} \boldsymbol{w}_{s}, \boldsymbol{n}_{1}=-R_{e} S\left(\boldsymbol{p}_{s}\right) J_{s}^{-1} S\left(R_{e}^{\mathrm{T}}(\boldsymbol{\omega}-\right.$ $\left.\left.\omega_{e}\right)\right) J_{s} R_{e}^{\mathrm{T}}\left(\omega-\omega_{e}\right), n_{2}=J R_{e} J_{s}^{-1} S\left(R_{e}^{\mathrm{T}}\left(\omega-\omega_{e}\right)\right) J_{s} R_{e}^{\mathrm{T}}\left(\omega-\omega_{e}\right)$.

Similarly, the relative position model between points $\mathbf{H}$ and $\mathbf{P}^{\prime}$ shown in Figure 2 can be easily derived by

$$
\begin{gathered}
\dot{\boldsymbol{r}}_{e}^{\prime}=\boldsymbol{v}_{e}^{\prime}-S(\boldsymbol{\omega}) \boldsymbol{r}_{e}^{\prime} \\
\dot{\boldsymbol{v}}_{e}^{\prime}=-\boldsymbol{h}^{\prime}-g R^{\mathrm{T}} \boldsymbol{e}_{3}+\boldsymbol{d}_{1}^{\prime}+\boldsymbol{n}_{1}^{\prime}+\frac{T}{m} \boldsymbol{e}_{3}
\end{gathered}
$$

where $\boldsymbol{r}_{e}^{\prime}=\boldsymbol{r}-R_{e} \boldsymbol{r}_{p_{s}}^{\prime}, \boldsymbol{v}_{e}^{\prime}=\boldsymbol{v}-R_{e} \boldsymbol{v}_{p_{s}}^{\prime}, \boldsymbol{r}_{p_{s}}^{\prime}=\boldsymbol{r}_{s}+\boldsymbol{p}_{s}^{\prime}, \boldsymbol{v}_{p_{s}}^{\prime}=$ $\boldsymbol{v}_{s}+S\left(\omega_{s}\right) \boldsymbol{p}_{s}^{\prime}, \boldsymbol{h}^{\prime}=S(\boldsymbol{\omega}) \boldsymbol{v}_{e}^{\prime}+S^{2}\left(\boldsymbol{\omega}-\omega_{e}\right) R_{e} \boldsymbol{p}_{s}^{\prime}, \boldsymbol{d}_{1}^{\prime}=\frac{\boldsymbol{d}_{h}}{m}-\frac{R_{e} \boldsymbol{d}_{s}}{m m_{s}}+$ $\frac{1}{m} R_{e} S\left(\boldsymbol{p}_{s}^{\prime}\right) J_{s}^{-1} \boldsymbol{w}_{s}, \boldsymbol{n}_{1}^{\prime}=-R_{e} S\left(\boldsymbol{p}_{s}^{\prime}\right) J_{s}^{-1} S\left(R_{e}^{\mathrm{T}}\left(\boldsymbol{\omega}-\boldsymbol{\omega}_{e}\right)\right) J_{s} R_{e}^{\mathrm{T}}\left(\boldsymbol{\omega}-\boldsymbol{\omega}_{e}\right)$.

Remark 3. Since the classical quadrotor tracking controller design only focuses on the dynamics of quadrotor, and the desired motion trajectory is generally simplified as the known differentiable functions, so that the tracking error system ignores the dynamical property of the target systems, so the modeling and control are not suitable for the two vehicles coordinated control missions, such as the shipboard landing operations in this study. From the equations (9)-(14), the mathematical model of the landing missions is strongly coupled and still underactuated. Specifically, the error model of pose motion is related with the inertia parameters of two vehicles, such that the dynamical model is very complicated with respect to the classical quadrotor tracking model, and the relative translational motion is coupled with the relative rotational motion.

Remark 4. Since the shipboard landing mission of the quadrotor is considered as autonomous task in this study, so that the motion information of the quadrotor and relative motion information between two vehicles could be measured directly by the different sensors mounted on the quadrotor. Then, the measured information is easily represented in the body-fixed frame of the quadrotor. Thus, the relative model is described in the body fixed frame of the quadrotor to facilitate the measurement and calculations. Moreover, if the relative motion model is described in other frames, such as the Earth-centered inertial coordinate frame, then the control inputs should be transformed into the body-fixed frame of the quadrotor, thus the coordinate transformation of the control inputs for the quadrotor can be avoided when the relative motion model is described in the body-fixed frame of the quadrotor. 
Assumption 1. The mass and inertial matrices of the quadrotor and the ship are all known. The external disturbances are unknown bounded vectors so that $\left\|\boldsymbol{d}_{h}\right\| \leq \bar{d}_{h},\left\|\boldsymbol{w}_{h}\right\| \leq \bar{w}_{h}$, $\left\|\boldsymbol{d}_{s}\right\| \leq \bar{d}_{s}$, and $\left\|\boldsymbol{w}_{s}\right\| \leq \bar{w}_{s}$ with known constants $\bar{d}_{h}, \bar{w}_{h}, \bar{d}_{s}, \bar{w}_{s}$.

Assumption 2. The pose motion information of the quadrotor $\boldsymbol{r}, \boldsymbol{v}, \boldsymbol{\sigma}, \omega$ and the relative pose information $\boldsymbol{r}_{e}, \boldsymbol{v}_{e}, \sigma_{e}, \omega_{e}$ between two vehicles are measured exactly by the measurement devices mounted on the bodies of quadrotor [10]-[14].

The control objective of the autonomous position approaching phase is to design the quadrotor's control inputs $T$ and $\tau$ so that position tracking error $\boldsymbol{r}_{e}$ ultimately converges to the small neighborhood of zero. Then, the control objective of the autonomous vertical landing phase is to design the quadrotor's control inputs $T$ and $\tau$ so that relative height $r_{e 3}^{\prime}$ and relative attitude $\sigma_{e}$ ultimately converge to the small neighborhood of zero, where $r_{e 3}^{\prime}$ is the third entry of vector $\boldsymbol{r}_{e}^{\prime}$. Thus the quadrotor shipboard landing missions can be achieved successfully.

\section{Controller Design}

\subsection{Controller Design for Position Approaching Phase}

Based on the model of (1), (9), and (10), the control inputs $T$ and $\tau$ of the quadrotor can be derived based on under-actuated control system design. Thus the outer-loop position controller design and inner-loop attitude controller are developed in the autonomous position approaching phase, so that the quadrotor tracks the desired position $\mathbf{P}$ in Figure 1 to achieve the position approaching mission, when the initial position of the quadrotor is far away from the ship deck.

Consider the relative position model in (9) and (10). Define the virtual position error $\overline{\boldsymbol{r}}_{e}=R \boldsymbol{r}_{e}-\boldsymbol{\eta}$ and virtual velocity error $\overline{\boldsymbol{v}}_{e}=R \boldsymbol{v}_{e}-\boldsymbol{\alpha}-\dot{\boldsymbol{\eta}}$ with an auxiliary state $\boldsymbol{\eta}$ and virtual control input $\boldsymbol{\alpha}$. Then,

$$
\dot{\overline{\boldsymbol{r}}}_{e}=R S(\omega) \boldsymbol{r}_{e}+R \boldsymbol{v}_{e}-R S(\omega) \boldsymbol{r}_{e}-\dot{\boldsymbol{\eta}}=\overline{\boldsymbol{v}}_{e}+\boldsymbol{\alpha}
$$

Choose a Lyapunov function candidate $V_{1}=\frac{1}{2} \overline{\boldsymbol{r}}_{e}^{\mathrm{T}} \overline{\boldsymbol{r}}_{e}$. Then the time derivative of $V_{1}$ based on (15) is $\dot{V}_{1}=\overline{\boldsymbol{r}}_{e}^{\mathrm{T}}\left(\overline{\boldsymbol{v}}_{e}+\boldsymbol{\alpha}\right)$. Design the virtual control input

$$
\boldsymbol{\alpha}=-k_{1} \overline{\boldsymbol{r}}_{e}
$$

where $k_{1}>0$. Substituting (16) into (15) yields

$$
\dot{\overline{\boldsymbol{r}}}_{e}=-k_{1} \overline{\boldsymbol{r}}_{e}+\overline{\boldsymbol{v}}_{e}
$$

Moreover, the time derivative of $V_{1}$ satisfies

$$
\dot{V}_{1}=-k_{1} \overline{\boldsymbol{r}}_{e}^{\mathrm{T}} \overline{\boldsymbol{r}}_{e}+\overline{\boldsymbol{r}}_{e}^{\mathrm{T}} \overline{\boldsymbol{v}}_{e}
$$

Furthermore, from (10), one has

$$
\begin{aligned}
\dot{\overline{\boldsymbol{v}}}_{e}= & R S(\boldsymbol{\omega}) \boldsymbol{v}_{e}-R \boldsymbol{h}-g \boldsymbol{e}_{3}+R \boldsymbol{d}_{1} \\
& +R \boldsymbol{n}_{1}+\frac{T}{m} \boldsymbol{R e}_{3}-\dot{\boldsymbol{\alpha}}-\ddot{\boldsymbol{\eta}}
\end{aligned}
$$

Choose a Lyapunov function candidate

$$
V_{2}=V_{1}+\frac{1}{2} \overline{\boldsymbol{v}}_{e}^{\mathrm{T}} \overline{\boldsymbol{v}}_{e}
$$

Then the time derivative of $V_{2}$ based on (19) is

$$
\begin{aligned}
\dot{V}_{2}= & -k_{1} \overline{\boldsymbol{r}}_{e}^{\mathrm{T}} \overline{\boldsymbol{r}}_{e}+\overline{\boldsymbol{v}}_{e}^{\mathrm{T}}\left(\overline{\boldsymbol{r}}_{e}+R S(\boldsymbol{\omega}) \boldsymbol{v}_{e}-R \boldsymbol{h}\right. \\
& \left.-g \boldsymbol{e}_{3}+R \boldsymbol{d}_{1}+R \boldsymbol{n}_{1}+\frac{T}{m} \operatorname{Re}_{3}-\dot{\boldsymbol{\alpha}}-\ddot{\boldsymbol{\eta}}\right)
\end{aligned}
$$

Since $\overline{\boldsymbol{v}}_{e}^{\mathrm{T}} \boldsymbol{R} \boldsymbol{d}_{1} \leq \bar{d}_{1}\left\|R\left|\left\|\left|\overline{\boldsymbol{v}}_{e} \| \leq \sum_{i=1}^{3} \bar{d}_{1}\right| \bar{v}_{e i} \mid \leq \sum_{i=1}^{3} \bar{d}_{1}\left[\bar{v}_{e i} \tanh \left(\frac{\bar{v}_{e i}}{\epsilon}\right)+\right.\right.\right.\right.$ $\kappa \epsilon]=\bar{d}_{1} \overline{\boldsymbol{v}}_{e}^{\mathrm{T}} \tanh \left(\frac{\bar{v}_{e}}{\epsilon}\right)+3 \kappa \epsilon$, where $\bar{d}_{1}=\bar{d}_{h}+\frac{\bar{d}_{s}}{m_{s}}+\bar{w}_{s}\left\|\boldsymbol{p}_{s}\right\|\left\|J_{s}^{-1}\right\|$, $\epsilon>0, \kappa=0.2785$. Then,

$$
\begin{aligned}
\dot{V}_{2} \leq & -k_{1} \overline{\boldsymbol{r}}_{e}^{\mathrm{T}} \overline{\boldsymbol{r}}_{e}+\overline{\boldsymbol{v}}_{e}^{\mathrm{T}}\left[\overline{\boldsymbol{r}}_{e}+R S(\boldsymbol{\omega}) \boldsymbol{v}_{e}-R \boldsymbol{h}-g \boldsymbol{e}_{3}\right. \\
& \left.+\bar{d}_{1} \tanh \left(\frac{\overline{\boldsymbol{v}}_{e}}{\epsilon}\right)+R \boldsymbol{n}_{1}+\frac{T}{m} \boldsymbol{R e}_{3}-\dot{\boldsymbol{\alpha}}-\ddot{\boldsymbol{\eta}}\right]+3 \kappa \epsilon
\end{aligned}
$$

Introduce an auxiliary system

$$
\ddot{\boldsymbol{\eta}}=-\beta_{1} \tanh \left(l_{1} \boldsymbol{\eta}+l_{2} \dot{\boldsymbol{\eta}}\right)-\beta_{2} \tanh \left(l_{2} \dot{\boldsymbol{\eta}}\right)+\boldsymbol{\alpha}_{\eta}
$$

where $\boldsymbol{\alpha}_{\eta}=k_{2} \overline{\boldsymbol{v}}_{e}-\dot{\boldsymbol{\alpha}}+\overline{\boldsymbol{r}}_{e}+R S(\boldsymbol{\omega}) \boldsymbol{v}_{e}-R \boldsymbol{h}+R \boldsymbol{n}_{1}, k_{2}>0$, $\beta_{i}>0, l_{i}>0(i=1,2)$, and $\boldsymbol{\eta}(0)=\dot{\boldsymbol{\eta}}(0)=\mathbf{0}$.

Remark 5. In the auxiliary system (23), the signal $\dot{\alpha}$ is difficult to directly calculate the time derivative of $\boldsymbol{\alpha}$ from (16), since the $\dot{\omega}$ is unknown. However, as shown in [31], a second-order command filter $\ddot{\boldsymbol{y}}=-2 \xi \omega_{n} \dot{\boldsymbol{y}}-\omega_{n}^{2}(\boldsymbol{y}-\boldsymbol{\alpha})$ with input $\boldsymbol{\alpha}$ and output $\dot{\boldsymbol{y}}$ under the initial conditions $\boldsymbol{y}(0)=\boldsymbol{\alpha}(0)$ and $\dot{\boldsymbol{y}}(0)=\mathbf{0}$ can be employed in practice to achieve high-precision estimation of $\dot{\boldsymbol{\alpha}}$ by setting the suitable filter parameters $\xi$ and $\omega_{n}$.

Design the control input

$$
\begin{aligned}
\boldsymbol{u}=\frac{T}{m} R_{d} \boldsymbol{e}_{3}= & -\beta_{1} \tanh \left(l_{1} \boldsymbol{\eta}+l_{2} \dot{\boldsymbol{\eta}}\right)-\beta_{2} \tanh \left(l_{2} \dot{\boldsymbol{\eta}}\right) \\
& +g \boldsymbol{e}_{3}-\bar{d}_{1} \tanh \left(\frac{\overline{\boldsymbol{v}}_{e}}{\epsilon}\right)
\end{aligned}
$$

where $R_{d}$ is the desired attitude for inner-loop attitude motion of the quadrotors. Then the desired thrust force is

$$
T=m\|\boldsymbol{u}\|
$$

Thus, from (22), one has

$$
\dot{V}_{2} \leq-k_{1}\left\|\overline{\boldsymbol{r}}_{e}\right\|^{2}-k_{2}\left\|\overline{\boldsymbol{v}}_{e}\right\|^{2}+\overline{\boldsymbol{v}}_{e}^{\mathrm{T}} \frac{T}{m} \bar{R}_{e} \boldsymbol{e}_{3}+3 \kappa \epsilon
$$

where $\bar{R}_{e}=R_{d}-R$.

From the system model in (1), we find that the transition matrix $R$ and control thrust $T$ will affect the translational motion of the quadrotors. Notice from (1) that the transition matrix $R$ in terms of MRP can also be regarded as the output of the rotational subsystem. This can be considered as the cascade property of the quadrotors. A hierarchical design strategy is introduced to implement the control system. Consequently, translational and rotational controllers can be designed separately. The translational controller is firstly designed to extract the desired thrust force $T$ and attitude matrix $R_{d}$. The desired attitude information can enable a quadrotor to track the desired position trajectory. Thereafter, the desired torque vector $\tau$ is determined by the rotational controller with the desired attitude matrix. At last, $T$ and $\tau$ can be treated as the input for the actuators to implement 
the whole control system. Then from (24), the desired MRP attitude for the inner-loop attitude motion of the quadrotor can be extracted by [32]

$$
\sigma_{d}=\frac{1}{2 T_{t} \sigma_{t}\left(1+\sigma_{t}\right)}\left[u_{2},-u_{1}, 0\right]^{\mathrm{T}}
$$

where $T_{t}=\frac{T}{m}, \sigma_{t}=\sqrt{\frac{u_{3}}{2 T_{t}}+\frac{1}{2}}$, and $u_{i}(i=1,2,3)$ is the $i$ th entry of the vector $\boldsymbol{u}$. To avoid the singularities of the thrust force and desired attitude, the following constraint must hold

$$
\sqrt{3}\left(\beta_{1}+\beta_{2}+\frac{\bar{d}_{1}}{m}\right)<g
$$

Remark 6. Since the thrust force $T$ should satisfy $T \geq 0$, thus the hyperbolic tangent function and conditions (28) are employed to guarantee the bounded property of thrust force such that $g-\sqrt{3}\left(\beta_{1}+\beta_{2}+\frac{\bar{d}_{1}}{m}\right) \leq\|\boldsymbol{u}\| \leq g+\sqrt{3}\left(\beta_{1}+\beta_{2}+\frac{\bar{d}_{1}}{m}\right)$ always holds. According to (25), (28), and $m>0$, we can obtain $\boldsymbol{u} \neq \mathbf{0}$ and $T>0$ instead of $T \geq 0$, thus the singularity of the thrust force $T$ can be avoided and the singularity of the desired attitude $\sigma_{d}$ is also avoided from (27).

Since the first and second time-derivatives of desired attitude $\sigma_{d}$ cannot be derived analytically, a stable second-order command filter is employed by

$$
\dot{\boldsymbol{x}}_{1}=\boldsymbol{x}_{2}, \dot{\boldsymbol{x}}_{2}=-2 \xi \omega_{n} \boldsymbol{x}_{2}-\omega_{n}^{2}\left(\boldsymbol{x}_{1}-\boldsymbol{\sigma}_{d}\right)
$$

with states $\boldsymbol{x}_{1}, \boldsymbol{x}_{2}$, and input $\boldsymbol{\sigma}_{d}$, where $\xi$ and $\omega_{n}$ are the damping ratio and frequency, respectively. The initial conditions for (29) are set as $\boldsymbol{x}_{1}(0)=\sigma_{d}(0)$ and $\boldsymbol{x}_{2}(0)=\mathbf{0}$, and the output of the command filter (29) is defined as

$$
\boldsymbol{\sigma}_{c} \triangleq \boldsymbol{x}_{1}, \quad \dot{\boldsymbol{\sigma}}_{c} \triangleq \boldsymbol{x}_{2}, \quad \ddot{\boldsymbol{\sigma}}_{c} \triangleq-2 \xi \omega_{n} \boldsymbol{x}_{2}-\omega_{n}^{2}\left(\boldsymbol{x}_{1}-\boldsymbol{\sigma}_{d}\right)
$$

Remark 7. It should be pointed out that the command attitude trajectory $\sigma_{c}$ is generated by command filter to approximate the desired attitude $\sigma_{d}$, and the errors between $\sigma_{c}$ and $\sigma_{d}$ can be reduced by choosing larger parameters $\omega_{n}$ in (29), but its robustness for high-frequency disturbances would be reduced significantly.

Instead of tracking $\sigma_{d}$ directly, we let the attitude subsystem track $\sigma_{c}$. By using $\sigma_{c}, \dot{\sigma}_{c}$ and $\ddot{\sigma}_{c}$ in (30), we can derive the command angular velocity $\omega_{c}$ and the command angular acceleration $\dot{\omega}_{c}$ as $[33,34]$

$$
\omega_{c}=G^{-1}\left(\sigma_{c}\right) \dot{\boldsymbol{\sigma}}_{c}, \dot{\omega}_{c}=\dot{G}^{-1}\left(\sigma_{c}\right) \dot{\boldsymbol{\sigma}}_{c}+G\left(\sigma_{c}\right) \ddot{\boldsymbol{\sigma}}_{c}
$$

where $\dot{G}^{-1}\left(\sigma_{c}\right)=-G^{-1}\left(\sigma_{c}\right) \dot{G}\left(\sigma_{c}\right) G^{-1}\left(\sigma_{c}\right), G\left(\sigma_{c}\right)=\frac{1}{4}[(1-$ $\left.\left.\sigma_{c}^{\mathrm{T}} \sigma_{c}\right) I_{3}+2 S\left(\sigma_{c}\right)+2 \sigma_{c} \sigma_{c}^{\mathrm{T}}\right]$, and $\dot{G}\left(\sigma_{c}\right)=\frac{8}{\left(1+\sigma_{c}^{\mathrm{T}} \sigma_{c}\right)^{2}}\left[\dot{\boldsymbol{\sigma}_{c}} \boldsymbol{\sigma}_{c}^{\mathrm{T}}+\right.$ $\left.\boldsymbol{\sigma}_{c} \dot{\boldsymbol{\sigma}}_{c}^{\mathrm{T}}-\dot{\boldsymbol{\sigma}}_{c}^{\mathrm{T}} \boldsymbol{\sigma}_{c} I_{3}-S\left(\dot{\boldsymbol{\sigma}}_{c}\right)\right]-\frac{16 \sigma_{c}^{\mathrm{T}} \dot{\boldsymbol{\sigma}}_{c}}{\left(1+\boldsymbol{\sigma}_{c}^{\mathrm{T}} \boldsymbol{\sigma}_{c}\right)^{3}}\left[\left(1-\boldsymbol{\sigma}_{c}^{\mathrm{T}} \boldsymbol{\sigma}_{c}\right) I_{3}-2 S\left(\boldsymbol{\sigma}_{c}\right)+\right.$ $\left.2 \sigma_{c} \sigma_{c}^{\mathrm{T}}\right]$.

As long as command attitude trajectories $\sigma_{c}, \omega_{c}$, and $\dot{\omega}_{c}$ are obtained, the attitude error and angular velocity error expressed in $\mathcal{F}_{h}$ can be obtained as [35]

$$
\left\{\begin{array}{l}
\overline{\boldsymbol{\sigma}}_{e}=\frac{\left(1-\boldsymbol{\sigma}_{c}^{\mathrm{T}} \boldsymbol{\sigma}_{c}\right) \boldsymbol{\sigma}-\left(1-\boldsymbol{\sigma}^{\mathrm{T}} \boldsymbol{\sigma}\right) \boldsymbol{\sigma}_{c}-2 S\left(\boldsymbol{\sigma}_{c}\right) \boldsymbol{\sigma}}{1+\sigma^{\mathrm{T}} \boldsymbol{\sigma} \sigma_{c}^{\mathrm{T}} \boldsymbol{\sigma}_{c}+2 \sigma_{c}^{\mathrm{T}} \boldsymbol{\sigma}} \\
\overline{\boldsymbol{\omega}}_{e}=\omega-R_{e} \omega_{c}
\end{array}\right.
$$

where $\bar{R}_{e}=I_{3}-\frac{4\left(1-\overline{\boldsymbol{\sigma}}_{e}^{\mathrm{T}} \overline{\boldsymbol{\sigma}}_{e}\right) S\left(\overline{\boldsymbol{\sigma}}_{e}\right)}{\left(1+\overline{\boldsymbol{\sigma}}_{e}^{\mathrm{T}} \overline{\boldsymbol{\sigma}}_{e}\right)^{2}}+\frac{8 S^{2}\left(\overline{\boldsymbol{\sigma}}_{e}\right)}{\left(1+\overline{\boldsymbol{\sigma}}_{e}^{\mathrm{T}} \overline{\boldsymbol{\sigma}}_{e}\right)^{2}}$.

From (1) and (32), the attitude tracking error kinematics and dynamics are

$$
\left\{\begin{array}{l}
\dot{\bar{\sigma}}_{e}=G\left(\bar{\sigma}_{e}\right) \bar{\omega}_{e} \\
J \dot{\bar{\omega}}_{e}=J S\left(\bar{\omega}_{e}\right) \bar{R}_{e} \omega_{c}-S(\omega) J \omega-J R_{e} \dot{\omega}_{c}+\tau+w_{h}
\end{array}\right.
$$

with $G\left(\overline{\boldsymbol{\sigma}}_{e}\right)=\frac{1}{4}\left[\left(1-\overline{\boldsymbol{\sigma}}_{e}^{\mathrm{T}} \overline{\boldsymbol{\sigma}}_{e}\right) I_{3}+2 S\left(\overline{\boldsymbol{\sigma}}_{e}\right)+2 \overline{\boldsymbol{\sigma}}_{e} \overline{\boldsymbol{\sigma}}_{e}^{\mathrm{T}}\right]$.

Define a variable $\tilde{\boldsymbol{\omega}}_{e}=\overline{\boldsymbol{\omega}}_{e}-\boldsymbol{\zeta}$, where $\zeta$ is the virtual control input designed later. Then, from attitude kinematics in (33), one has

$$
\dot{\overline{\boldsymbol{\sigma}}}_{e}=G\left(\overline{\boldsymbol{\sigma}}_{e}\right)\left(\tilde{\boldsymbol{\omega}}_{e}+\zeta\right)
$$

Design the virtual controller $\zeta=-k_{3} \overline{\boldsymbol{\sigma}}_{e}$ and choose a Lyapunov function candidate $V_{3}=\frac{1}{2} \overline{\boldsymbol{\sigma}}_{e}^{\mathrm{T}} \overline{\boldsymbol{\sigma}}_{e}$. Then (34) and the time derivative of $V_{3}$ are rewritten as

$$
\begin{gathered}
\dot{\overline{\boldsymbol{\sigma}}}_{e}=G\left(\overline{\boldsymbol{\sigma}}_{e}\right)\left(\tilde{\boldsymbol{\omega}}_{e}-k_{3} \overline{\boldsymbol{\sigma}}_{e}\right) \\
\dot{V}_{3}=-k_{3} \overline{\boldsymbol{\sigma}}_{e}^{\mathrm{T}} G\left(\overline{\boldsymbol{\sigma}}_{e}\right) \overline{\boldsymbol{\sigma}}_{e}+\overline{\boldsymbol{\sigma}}_{e}^{\mathrm{T}} G\left(\overline{\boldsymbol{\sigma}}_{e}\right) \tilde{\boldsymbol{\omega}}_{e}
\end{gathered}
$$

where $k_{3}>0$. Considering the attitude tracking dynamics in (33) and virtual controller $\zeta$ yields

$$
\begin{aligned}
J \dot{\tilde{\omega}}_{e}= & J S\left(\overline{\boldsymbol{\omega}}_{e}\right) \bar{R}_{e} \omega_{c}-S(\omega) J \omega \\
& -J R_{e} \dot{\boldsymbol{\omega}}_{c}+\tau+\boldsymbol{w}_{h}-k_{3} J \dot{\bar{\sigma}}_{e}
\end{aligned}
$$

Choose the Lyapunov function candidate

$$
V_{4}=V_{3}+\frac{1}{2} \tilde{\omega}_{e}^{\mathrm{T}} J \tilde{\omega}_{e}
$$

Then, based on the fact $\tilde{\boldsymbol{\omega}}_{e}^{\mathrm{T}} \boldsymbol{w}_{h} \leq \sum_{i=1}^{3} \bar{w}_{h}\left[\tilde{\boldsymbol{\omega}}_{e i} \tanh \left(\frac{\tilde{\omega}_{e i}}{\epsilon}\right)+\kappa \epsilon\right]=$ $\bar{w}_{h} \tilde{\omega}_{e}^{\mathrm{T}} \tanh \left(\frac{\tilde{\omega}_{e}}{\epsilon}\right)+3 \kappa \epsilon$, the time derivative of $V_{4}$ is

$$
\begin{aligned}
\dot{V}_{4} \leq & -k_{3} \overline{\boldsymbol{\sigma}}_{e}^{\mathrm{T}} G\left(\overline{\boldsymbol{\sigma}}_{e}\right) \overline{\boldsymbol{\sigma}}_{e}+\tilde{\boldsymbol{\omega}}_{e}^{\mathrm{T}}\left[G^{\mathrm{T}}\left(\overline{\boldsymbol{\sigma}}_{e}\right) \overline{\boldsymbol{\sigma}}_{e}\right. \\
& +J S\left(\overline{\boldsymbol{\omega}}_{e}\right) \bar{R}_{e} \omega_{c}-S(\omega) J \boldsymbol{\omega}-J R_{e} \dot{\boldsymbol{\omega}}_{c} \\
& \left.+\boldsymbol{\tau}+\bar{w}_{h} \tanh \left(\frac{\tilde{\boldsymbol{\omega}}_{e}}{\epsilon}\right)-k_{3} J \dot{\overline{\boldsymbol{\sigma}}}_{e}\right]+3 \kappa \epsilon
\end{aligned}
$$

Then design the inner-loop attitude tracking controller of the quadrotor is

$$
\begin{aligned}
\boldsymbol{\tau}= & -k_{4} \tilde{\boldsymbol{\omega}}_{e}-G^{\mathrm{T}}\left(\overline{\boldsymbol{\sigma}}_{e}\right) \overline{\boldsymbol{\sigma}}_{e}-J S\left(\overline{\boldsymbol{\omega}}_{e}\right) \bar{R}_{e} \omega_{c}+S(\omega) J \omega \\
& +J R_{e} \dot{\boldsymbol{\omega}}_{c}+k_{3} J \dot{\overline{\boldsymbol{\sigma}}}_{e}-\bar{w}_{h} \tanh \left(\frac{\tilde{\boldsymbol{\omega}}_{e}}{\epsilon}\right)
\end{aligned}
$$

where $k_{4}>0$. Thus, from the fact $\frac{1}{4} \leq\left\|G\left(\overline{\boldsymbol{\sigma}}_{e}\right)\right\| \leq \frac{1}{2}$, (39) can be written as

$$
\dot{V}_{4} \leq-\frac{k_{3}}{4}\left\|\overline{\boldsymbol{\sigma}}_{e}\right\|^{2}-k_{4}\left\|\tilde{\boldsymbol{\omega}}_{e}\right\|^{2}+3 \kappa \epsilon
$$

\subsection{Controller Design for Vertical Landing Phase}

Based on the relative height model in (13) and (14), and the relative attitude model in (11) and (12), the control inputs $T$ and $\tau$ of the quadrotor can be derived based on the fully-actuated control system design approach. When the initial position of the quadrotor locates at point $\mathbf{P}$ shown in Figure 2, the controller design task is to propose control inputs $T$ and $\tau$ so that 
the height of the quadrotor is driven to the height of the terminal landing site $\mathbf{P}^{\prime}$ shown in Figure 2, while the attitude of the ship and the quadrotor are synchronized to successfully achieve the autonomous landing mission.

According to the structure of the relative position controller (24) with the auxiliary system (23), the height controller of the quadrotor in the autonomous vertical landing phase can be easily derived by

$$
\begin{aligned}
T= & \mid m \gamma_{1} \tanh \left(l_{1}^{\prime} \eta^{\prime}+l_{2}^{\prime} \dot{\eta}^{\prime}\right)-m \gamma_{2} \tanh \left(l_{2}^{\prime} \dot{\eta}^{\prime}\right) \\
& +m g-\bar{d}_{1} \tanh \left(\frac{\bar{v}_{e 3}^{\prime}}{\epsilon}\right) \mid
\end{aligned}
$$

with the following auxiliary system

$$
\ddot{\eta}^{\prime}=-\gamma_{1} \tanh \left(l_{1}^{\prime} \eta^{\prime}+l_{2}^{\prime} \dot{\eta}^{\prime}\right)-\gamma_{2} \tanh \left(l_{2}^{\prime} \dot{\eta}^{\prime}\right)+\alpha_{\eta}^{\prime}
$$

and expressions $\alpha_{\eta}^{\prime}=k_{6} \bar{v}_{e 3}^{\prime}-\dot{\alpha}^{\prime}+\bar{r}_{e 3}^{\prime}+f_{3}, \bar{r}_{e 3}^{\prime}=r_{m 3}-\eta^{\prime}$, $\bar{v}_{e 3}^{\prime}=v_{m 3}-\alpha^{\prime}-\dot{\eta}^{\prime}$, and $\alpha^{\prime}=-k_{5} \bar{r}_{e 3}^{\prime}$, where $\bar{r}_{e 3}^{\prime}, \bar{v}_{e 3}^{\prime}, f_{3}, r_{m 3}$, and $v_{m 3}$ are the third entries of the vectors $\overline{\boldsymbol{r}}_{e}^{\prime}, \overline{\boldsymbol{v}}_{e}^{\prime}, R S(\boldsymbol{\omega}) \boldsymbol{v}_{e}^{\prime}-R \boldsymbol{h}^{\prime}+$ $R n_{1}^{\prime}, R \boldsymbol{r}_{e}^{\prime}$, and $R \boldsymbol{v}_{e}^{\prime}$, respectively; $\boldsymbol{r}_{e}^{\prime}, \boldsymbol{v}_{e}^{\prime}, \boldsymbol{h}^{\prime}$, and $\boldsymbol{n}_{1}^{\prime}$ are derived from (13) and (14); the designing parameters $k_{5}>0, k_{6}>0$, $\gamma_{i}>0, l_{i}^{\prime}>0(i=1,2)$, and $\eta^{\prime}(0)=\dot{\eta}^{\prime}(0)=0$.

The attitude synchronization controller in the autonomous vertical landing phase can be designed based on backstepping technique from (11) and (12). Define variable $\omega_{m}=\omega_{e}-\chi$, where the virtual control input is $\chi=-k_{7} \sigma_{e}$. Then the time derivative of $\sigma_{e}$ is $\dot{\boldsymbol{\sigma}}_{e}=G\left(\sigma_{e}\right)\left(\omega_{m}-k_{7} \sigma_{e}\right)$. Choose the Lyapunov function candidate $V_{5}=\frac{1}{2} \sigma_{e}^{\mathrm{T}} \sigma_{e}$. Then the time derivative of $V_{5}$ is $\dot{V}_{5}=-k_{7} \sigma_{e}^{\mathrm{T}} G\left(\sigma_{e}\right) \sigma_{e}+\sigma_{e}^{\mathrm{T}} G\left(\sigma_{e}\right) \omega_{m}$. The time derivative of $\omega_{m}$ is $J \dot{\omega}_{m}=-S(\omega) J \omega-J S\left(\omega_{e}\right) \omega_{e}+n_{2}+\tau+$ $\boldsymbol{d}_{2}-k_{7} J \dot{\boldsymbol{\sigma}}_{e}$. Define the Lyapunov function candidate

$$
V_{6}=V_{5}+\frac{1}{2} \omega_{m}^{\mathrm{T}} J \omega_{m}
$$

Then, based on the fact $\omega_{m}^{\mathrm{T}} \boldsymbol{d}_{2} \leq \sum_{i=1}^{3} \bar{d}_{2}\left[\omega_{m i} \tanh \left(\frac{\omega_{m i}}{\epsilon}\right)+\kappa \epsilon\right]=$ $\bar{d}_{2} \omega_{m}^{\mathrm{T}} \tanh \left(\frac{\omega_{m}}{\epsilon}\right)+3 \kappa \epsilon$, the time derivative of $V_{6}$ is

$$
\begin{aligned}
\dot{V}_{6} \leq & -k_{7} \sigma_{e}^{\mathrm{T}} G\left(\sigma_{e}\right) \sigma_{e}+\omega_{m}^{\mathrm{T}}\left[G^{\mathrm{T}}\left(\sigma_{e}\right) \sigma_{e}-S(\omega) J \omega\right. \\
& \left.-J S\left(\omega_{e}\right) \omega_{e}+\boldsymbol{n}_{2}+\tau+\bar{d}_{2} \tanh \left(\frac{\omega_{m}}{\epsilon}\right)-k_{7} J \dot{\boldsymbol{\sigma}}_{e}\right]+3 \kappa \epsilon
\end{aligned}
$$

Then design the attitude synchronization controller as

$$
\begin{aligned}
\boldsymbol{\tau}= & -k_{8} \boldsymbol{\omega}_{m}-G^{\mathrm{T}}\left(\boldsymbol{\sigma}_{e}\right) \boldsymbol{\sigma}_{e}+S(\boldsymbol{\omega}) J \boldsymbol{\omega}+J S\left(\boldsymbol{\omega}_{e}\right) \boldsymbol{\omega}_{e} \\
& -\boldsymbol{n}_{2}+k_{7} J \dot{\boldsymbol{\sigma}}_{e}-\bar{w}_{h} \tanh \left(\frac{\boldsymbol{\omega}_{m}}{\epsilon}\right)
\end{aligned}
$$

where $k_{8}>0$. From the fact $\frac{1}{4} \leq\left\|G\left(\sigma_{e}\right)\right\| \leq \frac{1}{2}, \dot{V}_{6}$ satisfies

$$
\dot{V}_{6} \leq-\frac{k_{7}}{4}\left\|\sigma_{e}\right\|^{2}-k_{8}\left\|\omega_{m}\right\|^{2}+3 \kappa \epsilon
$$

Remark 8. For the dynamical model of relative position approaching phase including (9), (10), and the attitude dynamic$\mathrm{s}$ in (1), the proposed relative position approaching controller is constructed by the thrust input (25), torque input (40), auxiliary system (23), and command filter (29) with the positive tunable parameters $k_{1}, k_{2}, k_{3}, k_{4}, \beta_{1}, \beta_{2}, l_{1}, l_{2}, \xi, \omega_{n}$. Similarly, for the dynamical model of vertical landing phase including (11), (12), (13), and (14), the proposed vertical landing controller is constructed by the thrust input (42), torque input (45), and auxiliary system (43) with the positive tunable parameters $k_{5}, k_{6}, k_{7}, k_{8}, \gamma_{1}, \gamma_{2}, l_{1}^{\prime}, l_{2}^{\prime}$. Two controllers for two phases have the same structure with similar designing parameters, so that the tuning strategies of the designing parameters for two controllers are unified.

The structure of the control system is shown in Figure 3.

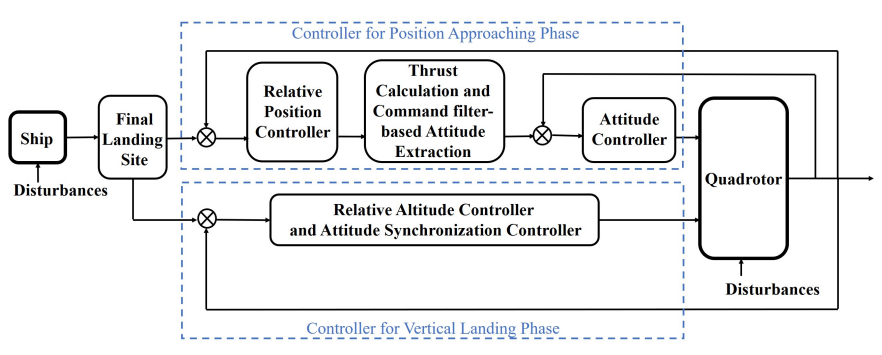

Figure 3: Block diagram of the closed-loop system for relative motion.

\section{Stability Analysis of Overall Systems}

Before the stability results, a significant lemma used in the stability analysis is shown firstly [36, 37].

Lemma 1. Consider the following nonlinear system

$$
\ddot{z}+a_{1} \tanh \left(a_{3} z+a_{4} \dot{z}\right)+a_{2} \tanh \left(a_{4} \dot{z}\right)-\boldsymbol{w}(t)=\mathbf{0}
$$

where $z \in \mathbb{R}^{n}, \boldsymbol{w}(t): \mathbb{R}^{+} \rightarrow \mathbb{R}^{n}$ is a time-varying vector, and $a_{i}(i=1,2,3,4)$ are positive constants. Denote $s=\left[a_{3} z^{\mathrm{T}}+\right.$ $\left.a_{4} \dot{z}^{\mathrm{T}}, a_{4} \dot{z}^{\mathrm{T}}\right]^{\mathrm{T}}$, if there exist $\bar{w}>0$ and $\bar{t}>0$ such that $\|\boldsymbol{w}(t)\|<\bar{w}$ for all $t \geq \bar{t}$, and the parameters $a_{5}=\frac{a_{3}}{a_{4}^{2}}, a_{2}$ and $a_{1}$ are set in sequence based on the rules $a_{5}>2 \bar{w}, a_{2}>\frac{2 a_{5} \bar{w}}{a_{5}-2 \bar{w}}, \sqrt{a_{5} a_{2}} \leq$ $a_{1} \leq \frac{1}{2}\left(a_{2}+a_{5}\right)$, then $z$ and $\dot{z}$ ultimately converge to the bounded set $\mathcal{Z}^{2}=\left\{\left[z^{\mathrm{T}}, \dot{z}^{\mathrm{T}}\right]^{\mathrm{T}} \in \mathbb{R}^{2 n}\|\mid \boldsymbol{s}\|<\bar{s}\right\}$, where $\bar{s}$ satisfies $\frac{a_{2}+a_{5}}{a_{2} a_{5} \bar{w}}<$ $\frac{\tanh ^{2}(\bar{s})}{\bar{s}}<\frac{1}{2}$.

Theorem 1. Consider the relative position dynamics (9), (10) and the quadrotor's attitude dynamics shown in the last two subequations of (1) under the Assumptions 1 and 2. Design the auxiliary system (23), control thrust (25), and control torque (40) for the quadrotor under the condition (28). Then the relative position $\boldsymbol{r}_{e}$ ultimately converges to the small neighborhood of zero by choosing suitable designing parameters.

Proof: Consider the Lyapunov function $V=V_{2}+V_{4}$. From (26) and (41), then the time derivative of $V$ satisfies $\dot{V} \leq-k_{1}\left\|\overline{\boldsymbol{r}}_{e}\right\|^{2}-k_{2}\left\|\overline{\boldsymbol{v}}_{e}\right\|^{2}-\frac{k_{3}}{4}\left\|\overline{\boldsymbol{\sigma}}_{e}\right\|^{2}-k_{4}\left\|\tilde{\boldsymbol{\omega}}_{e}\right\|^{2}+\overline{\boldsymbol{v}}_{e}^{\mathrm{T}} \frac{T}{m} \bar{R}_{e} \boldsymbol{e}_{3}+6 \kappa \epsilon$. Since $\left\|\frac{T}{m} \bar{R}_{e} \boldsymbol{e}_{3}\right\| \leq\|\boldsymbol{u}\|\left\|R_{d}-R\right\| \leq 2\left(\beta_{1}+\beta_{2}+g+\frac{\bar{d}_{1}}{m}\right) \triangleq \rho$ from (24) and (25), then $\dot{V} \leq-k_{e} V+\varpi$, where $k_{e}=\frac{\min \left\{k_{1}, \frac{k_{2}}{2}, \frac{k_{3}}{4}, k_{4}\right\}}{\frac{1}{2} \max \{1, \bar{\lambda}\}}, \varpi=$ $\frac{\rho^{2}}{2 k_{2}}+6 \kappa \epsilon, \bar{\lambda}$ is the maximum eigenvalue of matrix $J$. Then, it is easily shown that $\overline{\boldsymbol{r}}_{e}, \overline{\boldsymbol{v}}_{e}, \overline{\boldsymbol{\sigma}}_{e}, \tilde{\boldsymbol{\omega}}_{e}$ ultimately converge to the following bounded sets, $\Omega_{1}=\left\{\overline{\boldsymbol{r}}_{e}\left\|\mid \overline{\boldsymbol{r}}_{e}\right\| \leq \sqrt{\frac{2 \pi}{k_{e}}}\right\}, \Omega_{2}=\left\{\overline{\boldsymbol{v}}_{e}\left\|\overline{\boldsymbol{v}}_{e}\right\| \leq\right.$ 
$\left.\sqrt{\frac{2 \pi}{k_{e}}}\right\}, \Omega_{3}=\left\{\overline{\boldsymbol{\sigma}}_{e}\left\|\overline{\boldsymbol{\sigma}}_{e}\right\| \leq \sqrt{\frac{2 \sigma}{k_{e}}}\right\}, \Omega_{4}=\left\{\tilde{\boldsymbol{\omega}}_{e}\left\|\mid \tilde{\boldsymbol{\omega}}_{e}\right\| \leq \sqrt{\frac{2 \sigma}{\lambda k_{e}}}\right\}$, where $\lambda$ is the minimum eigenvalue of matrix $J$. Furthermore, from (16), and the definitions of the virtual position error $\overline{\boldsymbol{r}}_{e}=R \boldsymbol{r}_{e}-\boldsymbol{\eta}$ and virtual velocity error $\overline{\boldsymbol{v}}_{e}=R \boldsymbol{v}_{e}-\boldsymbol{\alpha}-\dot{\boldsymbol{\eta}}$, one has

$$
\left[\begin{array}{l}
\boldsymbol{r}_{e} \\
\boldsymbol{v}_{e}
\end{array}\right]=\bar{A}\left[\begin{array}{c}
\overline{\boldsymbol{r}}_{e} \\
\overline{\boldsymbol{v}}_{e}
\end{array}\right]+\bar{B}\left[\begin{array}{l}
\boldsymbol{\eta} \\
\dot{\boldsymbol{\eta}}
\end{array}\right]
$$

where $\bar{A}=\left[\begin{array}{ll}R^{\mathrm{T}} & O_{3} \\ -k_{1} R^{\mathrm{T}} & R^{\mathrm{T}}\end{array}\right]$ and $\bar{B}=\operatorname{diag}\left\{R^{\mathrm{T}}, R^{\mathrm{T}}\right\}$. Then, from (23) and Lemma 1, $\boldsymbol{\eta}$ and $\dot{\boldsymbol{\eta}}$ ultimately converge to the bounded set $\mathcal{Z}_{\eta}=\left\{\left[\boldsymbol{\eta}^{\mathrm{T}}, \dot{\boldsymbol{\eta}}^{\mathrm{T}}\right]^{\mathrm{T}} \in \mathbb{R}^{2 n} \mid\left\|\boldsymbol{s}_{\eta}\right\|<\bar{s}_{\eta}\right\}$, where $\boldsymbol{s}_{\eta}=$ $\left[l_{1} \boldsymbol{\eta}^{\mathrm{T}}+l_{2} \dot{\boldsymbol{\eta}}^{\mathrm{T}}, l_{2} \dot{\boldsymbol{\eta}}^{\mathrm{T}}\right]^{\mathrm{T}}$, and $\bar{s}_{\eta}$ satisfies $\frac{\beta_{2}+l_{3}}{\beta_{2} l_{3} \bar{\alpha}_{\eta}}<\frac{\tanh ^{2}\left(\bar{s}_{\eta}\right)}{\bar{s}_{\eta}}<\frac{1}{2}$ with $l_{3}=\frac{l_{1}}{l_{2}^{2}}$ and $\left\|\boldsymbol{\alpha}_{\eta}(t)\right\| \leq \bar{\alpha}_{\eta}$ for all $t \geq \bar{t}$. Therefore, from $\left\|R^{\mathrm{T}}\right\|=1,\|\bar{A}\| \leq \sqrt{6+3 k_{1}^{2}}$, and $\|\bar{B}\| \leq \sqrt{6}$, one has $\left\|\left[\boldsymbol{r}_{e}^{\mathrm{T}}, \boldsymbol{v}_{e}^{\mathrm{T}}\right]^{\mathrm{T}}\right\| \leq \sqrt{6+3 k_{1}^{2}}\left(\left\|\overline{\boldsymbol{r}}_{e}\right\|+\left\|\overline{\boldsymbol{v}}_{e}\right\|\right)+\sqrt{6}\left\|\left[\boldsymbol{\eta}^{\mathrm{T}}, \dot{\boldsymbol{\eta}}^{\mathrm{T}}\right]^{\mathrm{T}}\right\|$. Thus relative position $\boldsymbol{r}_{e}$ and relative velocity $\boldsymbol{v}_{e}$ ultimately converge to the bounded set

$$
\Omega=\left\{\left[\boldsymbol{r}_{e}^{\mathrm{T}}, \boldsymbol{v}_{e}^{\mathrm{T}}\right]^{\mathrm{T}} \mid\left\|\left[\boldsymbol{r}_{e}^{\mathrm{T}}, \boldsymbol{v}_{e}^{\mathrm{T}}\right]^{\mathrm{T}}\right\|<2 \sqrt{\frac{2\left(6+3 k_{1}^{2}\right) \varpi}{k_{e}}}+\sqrt{6} \bar{s}_{\eta}\right\} .
$$

This means that the ultimate bound of the relative position and relative velocity can be regulated to the small neighborhood of zero by choosing suitable designing parameters $k_{e}$ and $\bar{s}_{\eta}$.

Theorem 2. Consider the relative altitude dynamics in (13) and (14), and the relative attitude dynamics (11) and (12) under the Assumptions 1 and 2. Design the auxiliary system (43), control thrust (42), and control torque (45) for the quadrotor under the condition (28). Then the relative altitude $r_{e 3}^{\prime}$ and relative attitude $\sigma_{e}$ ultimately converge to the small neighborhoods of zero by choosing suitable designing parameters.

Proof: Consider the Lyapunov function $V^{\prime}=\frac{1}{2}\left[\left(\bar{r}_{e 3}^{\prime}\right)^{2}+\right.$ $\left.\left(\bar{v}_{e 3}^{\prime}\right)^{2}\right]+V_{6}$. Then the time derivative of $V^{\prime}$ is $\dot{V}^{\prime}=\bar{r}_{e 3}^{\prime} \dot{\bar{r}}_{e 3}^{\prime}+$ $\bar{v}_{e 3}^{\prime} \dot{\bar{v}}_{e 3}^{\prime}+\boldsymbol{\sigma}_{e}^{\mathrm{T}} \dot{\boldsymbol{\sigma}}_{e}+\boldsymbol{\omega}_{m}^{\mathrm{T}} J \dot{\boldsymbol{\omega}}_{m}$. From $\bar{r}_{e 3}^{\prime}=r_{m 3}-\eta^{\prime}$ and $\bar{v}_{e 3}^{\prime}=$ $v_{m 3}-\alpha^{\prime}-\dot{\eta}^{\prime}$, one has $\dot{\bar{r}}_{e 3}^{\prime}=\bar{v}_{e 3}^{\prime}+\alpha^{\prime}=-k_{5} \bar{r}_{e 3}^{\prime}+\bar{v}_{e 3}^{\prime}$ and $\dot{\bar{v}}_{e 3}^{\prime}=-f_{3}-g+d^{\prime}+\frac{T}{m}-\dot{\alpha}^{\prime}-\ddot{\eta}^{\prime}$, where $d^{\prime}$ denotes the third entry of the term $R d_{1}$. Then, from $\left|d^{\prime}\right| \leq \bar{d}_{1}$, (42), (43), and (46), one also has $\dot{V}^{\prime} \leq-k_{m} V^{\prime}+\varsigma$, where $k_{m}=\frac{\min \left\{k_{5}, \frac{k_{6}}{2}, \frac{k_{7}}{4}, k_{8}\right\}}{\frac{1}{2} \max \{1, \bar{\lambda}\}}$ and $\varsigma=\frac{\rho^{2}}{2 k_{6}}+$ $6 \kappa \epsilon$. Thus $\bar{r}_{e 3}, \bar{v}_{e 3}, \sigma_{e}, \omega_{m}$ ultimately converge to the following bounded sets, $\Omega_{5}=\left\{\bar{r}_{e 3}|| \bar{r}_{e 3} \mid \leq \sqrt{\frac{2 \varsigma}{k_{m}}}\right\}, \Omega_{6}=\left\{\bar{v}_{e 3}|| \bar{v}_{e 3} \mid \leq \sqrt{\frac{2 \varsigma}{k_{m}}}\right\}$, $\Omega_{7}=\left\{\sigma_{e}\left\|\mid \sigma_{e}\right\| \leq \sqrt{\frac{2 \varsigma}{k_{m}}}\right\}, \Omega_{8}=\left\{\omega_{m}\left\|\omega_{m}\right\| \leq \sqrt{\frac{2 \varsigma}{\underline{\lambda} k_{m}}}\right\}$. Thus, from $\bar{r}_{e 3}^{\prime}=r_{m 3}-\eta^{\prime}$ and $\bar{v}_{e 3}^{\prime}=v_{m 3}-\alpha^{\prime}-\dot{\eta}^{\prime}$, one has

$$
\boldsymbol{\rho}_{m} \triangleq\left[\begin{array}{l}
r_{m 3} \\
v_{m 3} \\
\boldsymbol{\sigma}_{e} \\
\boldsymbol{\omega}_{e}
\end{array}\right]=\bar{A}^{\prime}\left[\begin{array}{c}
\bar{r}_{e 3} \\
\bar{v}_{e 3} \\
\boldsymbol{\sigma}_{e} \\
\omega_{m}
\end{array}\right]+\bar{B}^{\prime}\left[\begin{array}{c}
\eta^{\prime} \\
\dot{\eta}^{\prime} \\
\mathbf{0} \\
\mathbf{0}
\end{array}\right]
$$

where $\bar{A}^{\prime}=\operatorname{diag}\left\{A_{1}^{\prime}, A_{2}^{\prime}\right\}, \quad \bar{B}^{\prime}=\operatorname{diag}\left\{I_{2}, A_{2}^{\prime}\right\}, \quad A_{1}^{\prime}=$ $\left[\begin{array}{ll}1 & 0 \\ -k_{5} & 1\end{array}\right], A_{2}^{\prime}=\left[\begin{array}{ll}I_{3} & O_{3} \\ -k_{7} I_{3} & I_{3}\end{array}\right]$. Then, one has $\left\|\boldsymbol{\rho}_{m}\right\| \leq$ $\sqrt{8+k_{5}^{2}+3 k_{7}^{2}}\left(\left|\bar{r}_{e 3}\right|+\left|\bar{v}_{e 3}\right|+\left\|\sigma_{e}\right\|+\left\|\omega_{m}\right\|\right)+\sqrt{8+3 k_{7}^{2}}\left\|\left[\eta^{\prime}, \dot{\eta}^{\prime}\right]^{\mathrm{T}}\right\|$. Since $r_{m 3}=\left[R_{31}, R_{32}, R_{33}\right] r_{e}^{\prime}$ and $v_{m 3}=\left[R_{31}, R_{32}, R_{33}\right] v_{e}^{\prime}$, where $R_{31}, R_{32}, R_{33}$ denote three elements of the third row in the rotation matrix $R$, then it is concluded from Lemma 1 that

$$
\Omega^{\prime}=\left\{\boldsymbol{\rho}_{m}^{\prime} \mid\left\|\boldsymbol{\rho}_{m}^{\prime}\right\|<4 \sqrt{\frac{2\left(8+k_{5}^{2}+3 k_{7}^{2}\right) \varsigma}{\min \{1, \underline{\lambda}\} k_{m}}}+\sqrt{8+3 k_{7}^{2}} \bar{s}_{\eta}^{\prime}\right\}
$$

based on $\left\|\left[R_{31}, R_{32}, R_{33}\right]\right\|=1$, where $\rho_{m}^{\prime} \triangleq\left[r_{e 3}^{\prime}, v_{e 3}^{\prime}, \sigma_{e}^{\mathrm{T}}, \omega_{e}^{\mathrm{T}}\right]^{\mathrm{T}}$, $\boldsymbol{s}_{\eta}^{\prime}=\left[l_{1}^{\prime} \eta^{\mathrm{T}}+l_{2}^{\prime} \dot{\eta}^{\mathrm{T}}, l_{2}^{\prime} \dot{\eta}^{\mathrm{T}}\right]^{\mathrm{T}}$, and $\bar{s}_{\eta}^{\prime}$ satisfies $\frac{\gamma_{2}+l_{3}^{\prime}}{\gamma_{2} l_{3}^{\prime} \bar{\alpha}_{\eta}^{\prime}}<\frac{\tanh ^{2}\left(\bar{s}_{\eta}^{\prime}\right)}{\bar{s}_{\eta}^{\prime}}<\frac{1}{2}$ with $l_{3}^{\prime}=\frac{l_{1}^{\prime}}{\left(l_{2}^{\prime}\right)^{2}}$ and $\left\|\alpha_{\eta}^{\prime}(t)\right\| \leq \bar{\alpha}_{\eta}^{\prime}$ for all $t \geq \bar{t}^{\prime}$. Thus relative altitude $r_{e 3}^{\prime}$ and relative attitude $\sigma_{e}$ ultimately converges to the small neighborhood of zero by choosing suitable designing parameters $k_{m}$ and $\bar{s}_{\eta}$.

Remark 9. Because of the under-actuated property of the drones, the completed mission is divided into two phases, so the switching condition is critical for the tracking and landing. Because the tracking and landing phases mainly related with the relative distance between two vehicles, so the landing phase is viewed as the relative disturbance should be relatively small from the tests in simulations. When the relative height of the drone and the ship deck is very small, the relative horizontal position errors of the vertical landing phase are acceptable in practical situation. If the relative height is relatively overlarge, then the relative horizontal position errors in landing phase will be quickly increased in the short time so that the landing phase is unsafe. Therefore the switching condition should be set based on the precisely relative-height measurement and the safety requirements of the relative horizontal position errors in final landing phase. This switching condition design and stability analysis of the overall system should be further studied in future.

\section{Simulations}

For validating the proposed control strategy, the smallscaled quadrotor and ship are employed in simulations. The parameters of the quadrotor and ship are listed here. The mass and inertia matrix of quadrotor are $m=7.4(\mathrm{~kg})$ and $J=\operatorname{diag}(0.16,0.3,0.31)\left(\mathrm{kgm}^{2}\right)$, respectively. The mass and inertia matrix of ship are $m_{s}=50(\mathrm{~kg})$ and $J_{s}=$ $\operatorname{diag}(30,20,30)\left(\mathrm{kgm}^{2}\right)$, respectively. The external force and torque of ship are assumed as $\boldsymbol{d}_{s}=[1,0.01,0.06 \sin (t)]^{T}(\mathrm{~N})$, $\boldsymbol{w}_{s}=[0.1 \sin (5 t), 0.1 \cos (1.5 t), 0.1 \sin (5 t)]^{T}(\mathrm{Nm})$.

In the relative position approaching phase, the external disturbances of the quadrotor are assumed as

$$
\boldsymbol{d}_{h}=\left[\begin{array}{c}
0.2 \sin (0.5 t)+0.25 \cos (0.5 t) \\
0.25 \sin (0.5 t)-0.2 \cos (0.5 t) \\
0.15 \sin (0.5 t)+0.15 \cos (0.5 t)
\end{array}\right](\mathrm{N})
$$

$\boldsymbol{w}_{h}=0.1 \boldsymbol{d}_{h}(\mathrm{Nm})$. The initial states of the quadrotor are set as $\boldsymbol{r}(0)=[10,10,8]^{T}(\mathrm{~m}), \boldsymbol{\sigma}(0)=[-0.002,0.002,0]^{T}, \boldsymbol{v}(0)=$ $\mathbf{0}(\mathrm{m} / \mathrm{s}), \boldsymbol{\omega}(0)=\mathbf{0}(\mathrm{rad} / \mathrm{s}), \boldsymbol{p}_{s}=[-1.5,0,1]^{T}(\mathrm{~m})$, and the initial states of the ship are set as zeros. The controller parameters 
are set as $k_{1}=1.2, k_{2}=3, k_{3}=0.5, k_{4}=15, \beta_{1}=0.6, \beta_{2}=$ $0.9, l_{1}=1.8, l_{2}=1, \omega_{n}=19, \zeta=1.2, \epsilon=0.05, \bar{d}=0.5, \bar{w}=$ 0.2 , and $\boldsymbol{\eta}(0)=\dot{\boldsymbol{\eta}}(0)=\mathbf{0}$.

In the vertical landing phase, to simulate the randomness of landing environment, the external disturbances of the quadrotor are assumed as

$$
\boldsymbol{d}_{h}=0.01\left[\begin{array}{c}
0.2 \sin (0.5 t)+0.25 \cos (0.5 t) \\
0.25 \sin (0.5 t)-0.2 \cos (0.5 t) \\
0.15 \sin (0.5 t)+0.15 \cos (0.5 t)
\end{array}\right]+0.5 \operatorname{Rand}(\mathrm{N})
$$

$\boldsymbol{w}_{h}=0.1 \boldsymbol{d}_{h}(\mathrm{Nm})$, and Rand denotes the three-dimensional normal distribution random function with zero mean and 0.05 variance to simulate the random disturbances. The vector $\boldsymbol{p}_{s}^{\prime}$ is set as $[-1.5,0,0.5]^{T}(\mathrm{~m})$. The initial pose of the quadrotor and ship are set the same as the final pose of relative position approaching phase: $\boldsymbol{r}(0)=[34.43,0.75,0.86]^{T}(\mathrm{~m}), \boldsymbol{\sigma}(0)=$ $[2.16,-8.4,-1.25]^{T} \times 10^{-4}, \boldsymbol{r}_{s}(0)=[35.98,-0.6,0.15]^{T}(\mathrm{~m})$, $\sigma_{s}(0)=[0.01,0.0012,0.01]^{T}$, and the initial linear and angular velocities of two vehicles are set as zeros. The controller parameters are set as $k_{5}=2, k_{6}=1, k_{7}=k_{8}=15, \gamma_{1}=$ $25, \gamma_{2}=5, l_{1}^{\prime}=0.5, l_{2}^{\prime}=0.7, \epsilon=0.2, \bar{d}=0.2, \bar{w}=0.1$, and $\eta^{\prime}(0)=\dot{\eta}^{\prime}(0)=0$.

Figures 3-9 show the simulation results of the relative position approaching phase using the proposed controller. The relative motion shown in Figure 3 illustrates that the quadrotor can approach directly above the ship and track the arbitrary threedimensional trajectory of the ship. This result also implies that the proposed controller can be applied on collaborative task scenarios. Figure 4 displays position trajectories of two vehicles, where the difference between two vehicles in $\mathrm{x}$ axis and $\mathrm{z}$ axis is owing to the distance between the origin $\mathbf{S}$ and the target point P. It can be seen in Figure 5 that relative position errors converge to zero within 20(s). Figure 6 and Figure 7 illustrate that the attitude motion of the quadrotor can track the desired MRP attitude of the inner-loop. Figure 8 and Figure 9 show control inputs of the quadrotor and external disturbances, respectively.

To highlight the advantage and effectiveness of the proposed method, we compared the proposed method with the nonadaptation version, because overcoming the impact of complex marine environments is one of keys for shipboard landing control. From the comparative simulation result in Figure 10, it is obvious that the adaptation law is beneficial to compensate for the impact of external disturbances. Figures 11-15 show the simulation results of the vertical landing phase based on the proposed controller. From Figure 11 and Figure 12, it can be observed that the vertical landing phase is completed after 1(s). At the landing time 1(s), the altitude and attitude of two vehicles are synchronized, and the relative horizontal position errors (refers to red hexagonal stars in Figure 13) are $r_{e 1}=-0.062(\mathrm{~m})$, $r_{e 2}=0.102(\mathrm{~m})$, which are inevitable and acceptable. Furthermore, as shown in Figure 13, we can conclude that the quadrotor should stop the engines before the time of 1(s) to achieve the final high-precision and safe landing mission, since the relative horizontal position errors would be largely increased if the quadrotors is still horizontally moving. Figures 14-16 show angular velocity of the quadrotor, control inputs of the quadrotor and external disturbances, respectively.
In order to satisfy the generality, we evaluated the control performance with different desired horizontal landing sites. The result shown in Figure 17 indicates that the proposed controllers can guarantee the successful and relatively precise shipboard landings. Therefore, it's reasonable to conclude that the proposed controllers are efficient enough to accomplish the autonomous shipboard landing operations despite the horizontal position errors between the final landing site and desired landing site. Furthermore, based on above theoretical analysis and simulation results, it can be claimed that the proposed twophases control system designing approach for the quadrotor shipboard landing missions is effective with acceptable control effort and transient response performance, while the stability and safety of shipboard landing operations are guaranteed.

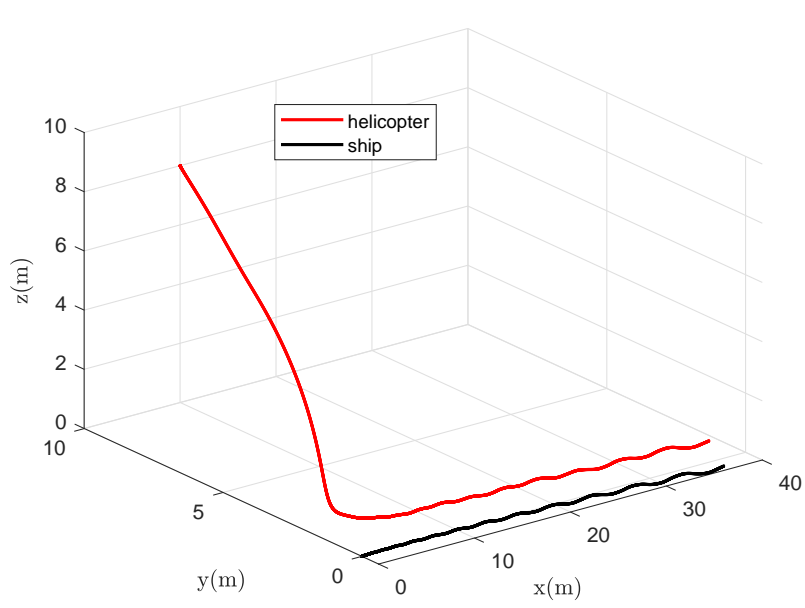

Figure 4: Three-dimensional relative motion of the position approaching phase.
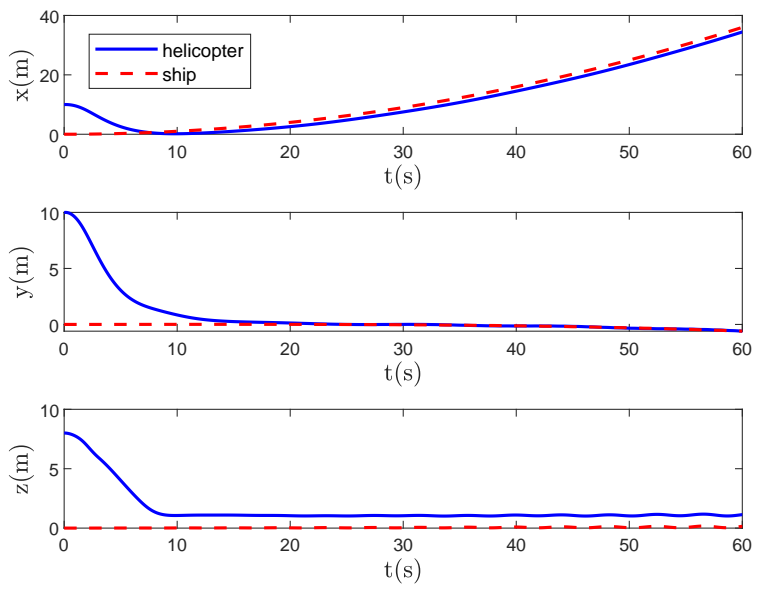

Figure 5: Position trajectory of two vehicles in the position approaching phase. 

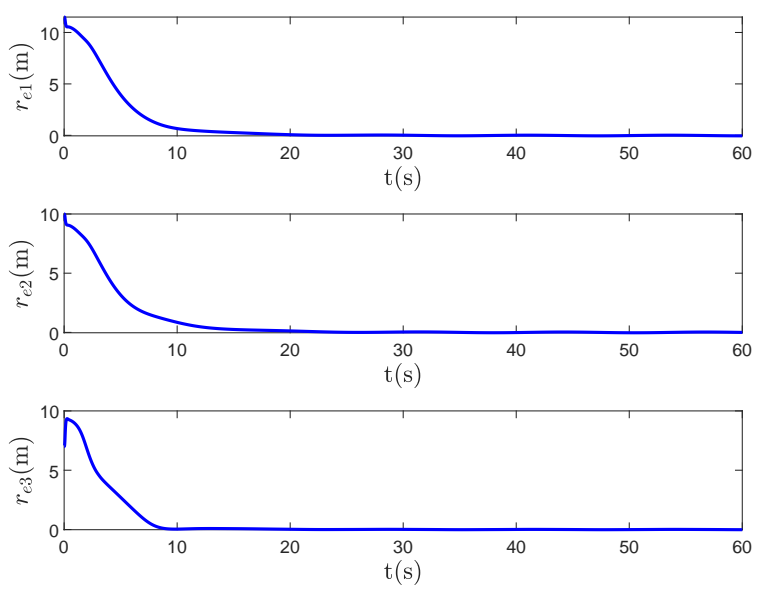

Figure 6: Relative position errors of the position approaching phase.
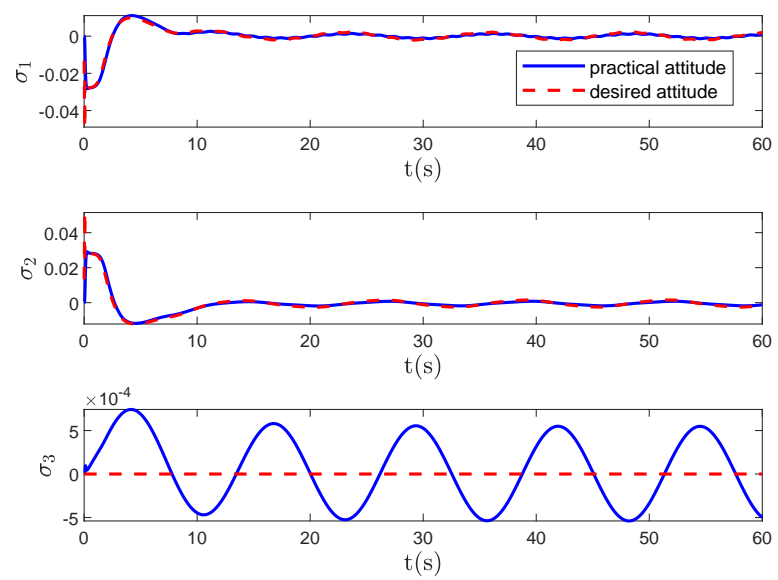

Figure 7: Attitude trajectory of the position approaching phase.
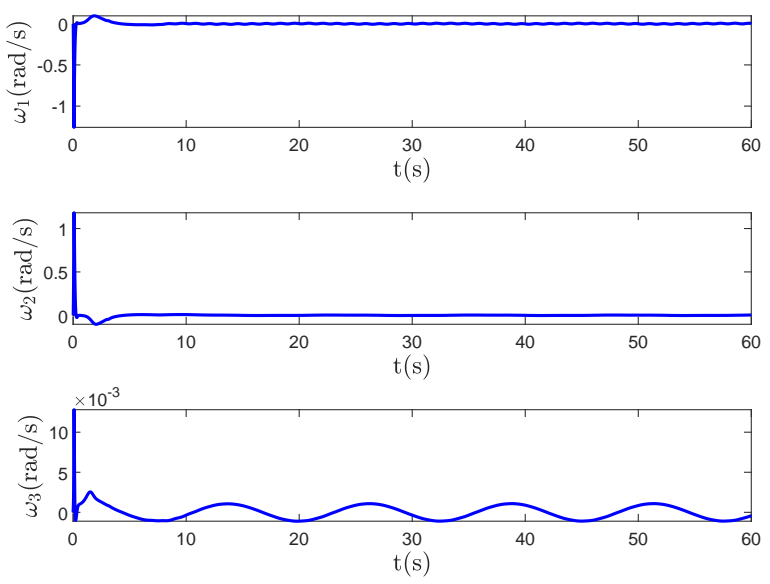

Figure 8: Angular velocity of the quadrotor in the position approaching phase.
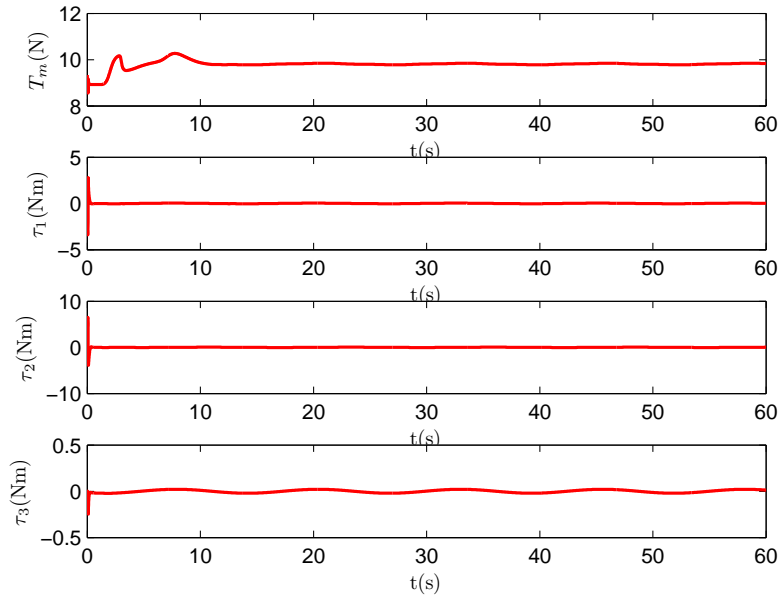

Figure 9: Control inputs of the quadrotor in the position approaching phase.
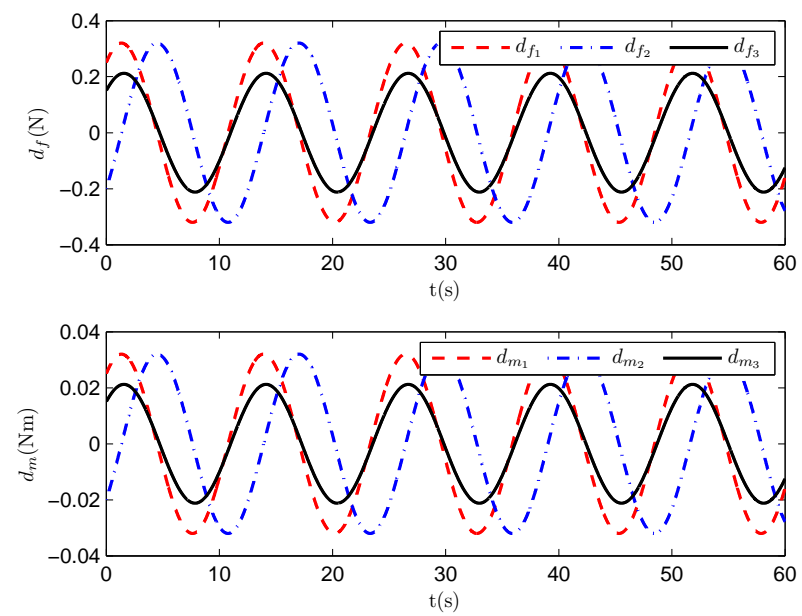

Figure 10: External disturbances in the position approaching phase.
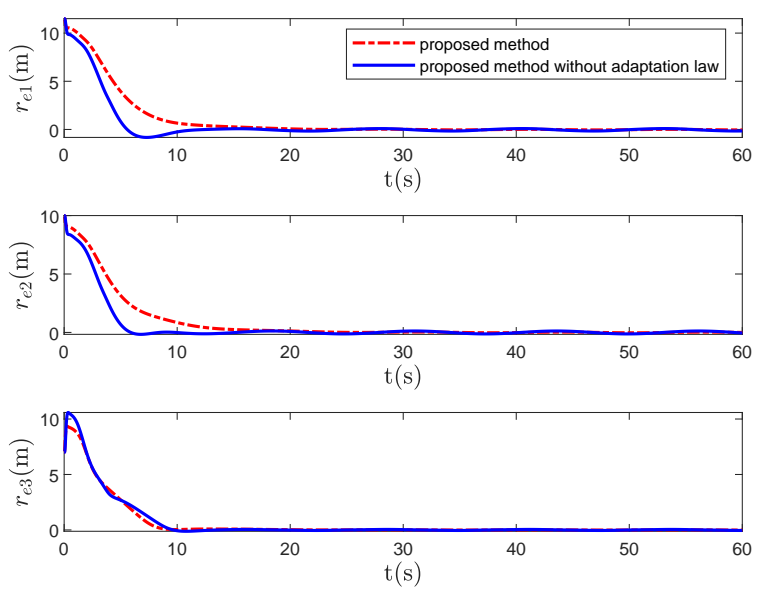

Figure 11: Comparison of relative position errors. 

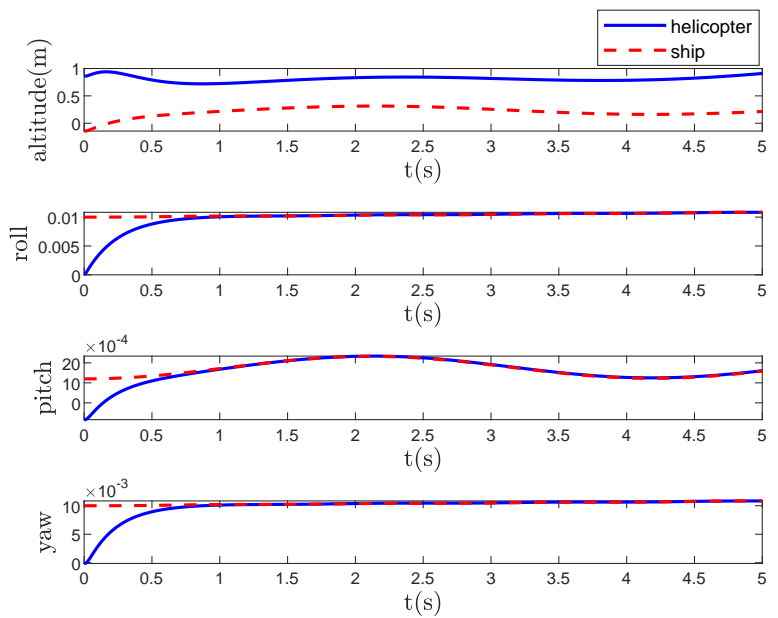

Figure 12: Attitude-altitude of two vehicles in the vertical landing phase.

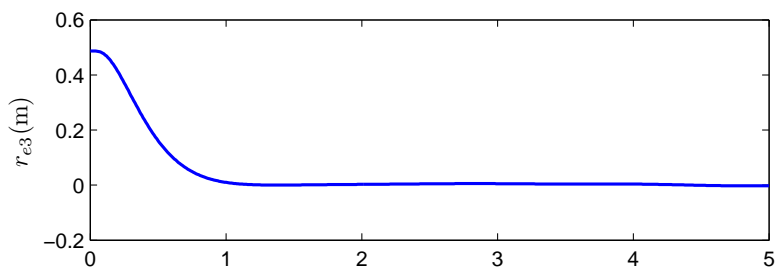

$\mathrm{t}(\mathrm{s})$

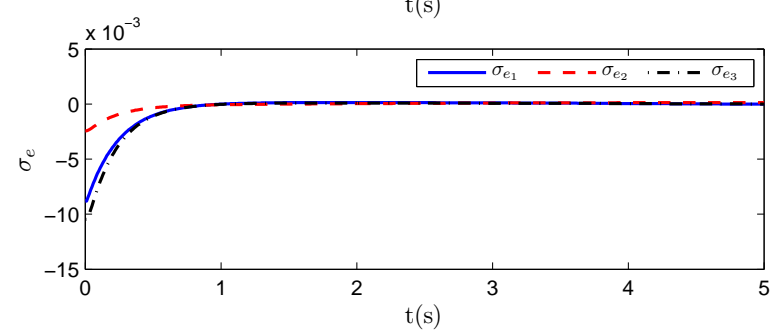

Figure 13: Relative attitude-altitude errors of the vertical landing phase.
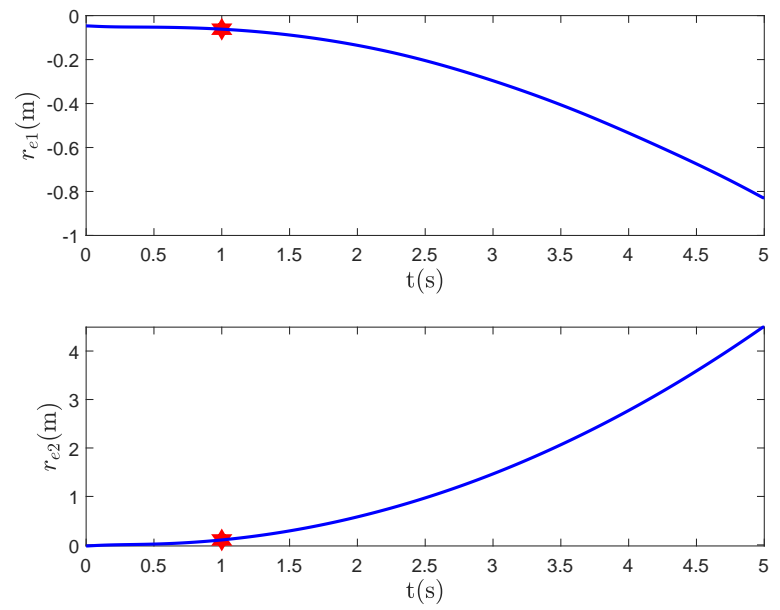

Figure 14: Relative horizontal position errors of the vertical landing phase.
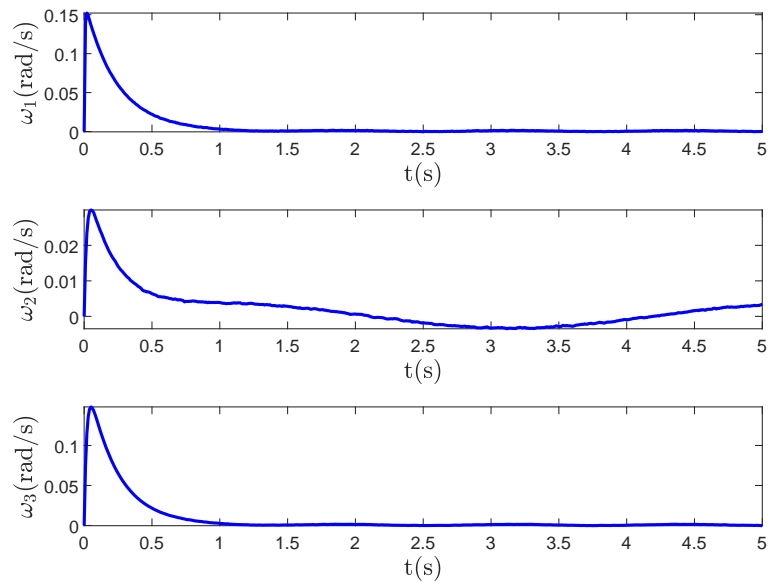

Figure 15: Angular velocity of the quadrotor in the vertical landing phase.
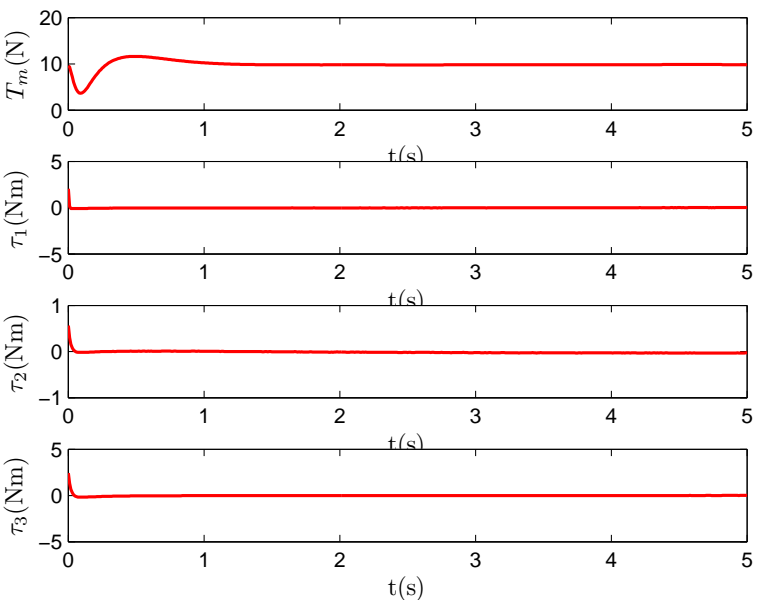

Figure 16: Control inputs of the quadrotor in the vertical landing phase.

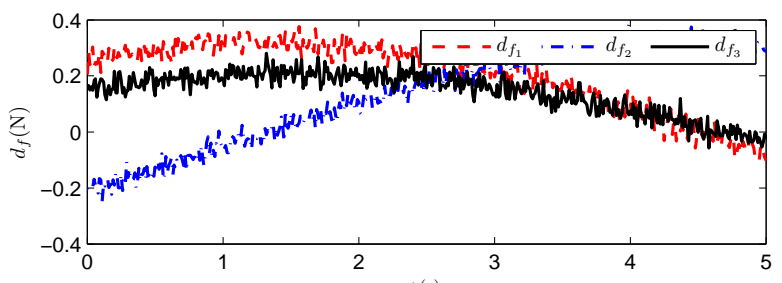

$t(s)$

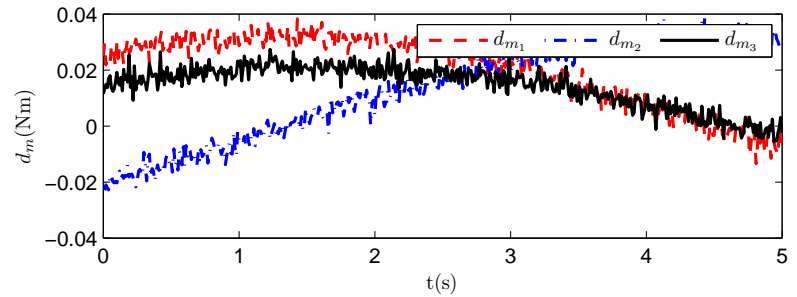

Figure 17: External disturbances in the vertical landing phase. 


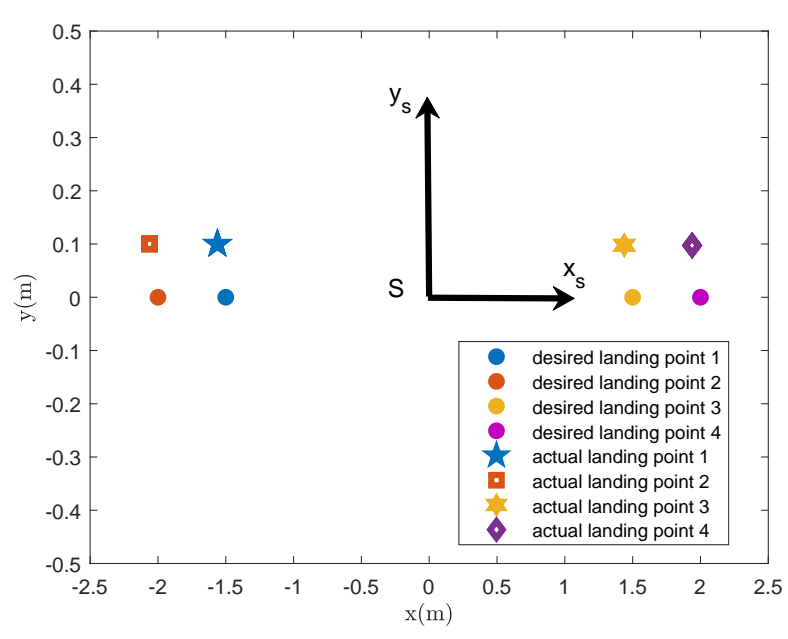

Figure 18: Horizontal final landing sites.

\section{Conclusion}

A novel two-phases control designing approach including the autonomous position approaching phase and the autonomous vertical landing phase for the shipboard landing mission is proposed in this study, where the dynamical model of relative motion between a quadrotor and a ship is derived with considering model uncertainties and the effect of landing site on the deck misaligned with the centre of mass of the ship. In particular, control singularity of the quadrotor is avoided by using the saturated force inputs and the bounded auxiliary system, while the second-order command filter is employed to avoid the complicated time derivative calculations. Theoretical analysis and simulation results verify that the proposed controller can ensure the ultimately uniformly convergence of tracking errors under suitable tuning parameters. Future works will focus on the control design and performance analysis for uncertain autonomous quadrotors working in dynamical transition process from autonomous position approaching phase to the autonomous vertical landing phase. Meanwhile, in order to ensure the safe maneuver of quadrotors, the pitch and roll angles of quadrotor should be constrained in prescribed values, thus the corresponding attitude constrained control problem will be further considered in future. Furthermore, the experiment tested on real quadrotor will be also the future works.

\section{Disclosure statement}

Statement of Contribution: Conceptualization, L. Sun; Methodology, L. Sun; Software, Y. Huang; Data curation, Y. Huang; Validation, Y. Huang; Formal Analysis, L. Sun and Z. Zheng; Visualization, Y. Huang and J. Jiang; Supervision, Z. Zheng, B. Zhu; Project Administration, Z. Zheng and B. Zhu; Funding Acquisition, L. Sun and Z. Zheng; Writing and Editing, L. Sun, Y. Huang, J. Jiang.

Funding: This work was supported by the National Natural Science Foundation of China [grant number 61903025]; the China Scholarship Council [grant number 201906465028]; the
Beijing Natural Science Foundation [grant number 4202038]; and the Fundamental Research Funds for the Central Universities [grant numbers FRF-BD-19-002A, FRF-GF-18-0028B].

Declarations of interest: The authors declare no conflict of interest.

\section{References}

[1] B. Zhu, W. Huo, Nonlinear control for a model-scaled helicopter with constraints on rotor thrust and fuselage attitude, Acta Autom. Sinica 40(11)(2014)2654-2664.

[2] T. Jiang, D. Lin, T. Song, Novel integral sliding mode control for smallscale unmanned helicopters, J. Franklin Inst. 356(5)(2019)2668-2689.

[3] Z. Zuo, S. Mallikarjunan, $L_{1}$ adaptive backstepping for robust trajectory tracking of UAVs, IEEE Trans. Ind. Electron. 64(4)(2017)2944-2954.

[4] B. Tian, H. Lu, Z. Zuo, Q. Zong, Y. Zhang, Multivariable finite-time output feedback trajectory tracking control of quadrotor helicopters, Int. J. Robust Nonlinear Control 28(1)(2018)281-295.

[5] D. Cabecinhas, R. Naldi, C. Silvestre, R. Cunha, L. Marconi, Robust landing and sliding maneuver hybrid controller for a quadrotor vehicle, IEEE Trans. Control Syst. Technol. 24(2)(2016)400-412.

[6] H. Shin, D. You, D. H. Shim, Autonomous shipboard landing algorithm for unmanned helicoters in crosswind, J. Intel. Robot. Syst. 74(2014)347361.

[7] S.-R. Oh, K. Paustubh, S. K. Agrawal, H. R. Pota, M. Garratt, Approaches for a tether-guided landing of an autonomous helicopter, IEEE Trans. Robotics 22(3)(2006)536-544.

[8] J. F. Horn, D. O. Bridges, D. Lee, Flight control design for alleviation of pilot workload during helicopter shipboard operations. Annual Forum Proc.-American Helicopter Society, American Helicopter Society Inc., 62(3)(2006)2032-2045.

[9] J. Blackwell, R. A. Feik, A mathematical model of the on-deck helicopter/ship dynamic interface, Aeronaut. Res. Lab. Aerodyn. Tech. Memorandum (1988)No. ARL-AERO-TM-405.

[10] Q. H. Truong, T. Rakotomamonjy, A. Taghizad, J. Biannic, Vision-based control for helicopter ship landing with handling qualities constraints, IFAC-PapersOnLine 49(17)(2016)118-123.

[11] W. Bagen, J. Hu, Y. Xu, A vision-based unmanned helicopter ship board landing system, 2nd Int. Congress Image and Signal Process. IEEE Publishing House (2009)1-5.

[12] S. Lin, M. A. Garratt, A. J. Lambert, Monocular vision-based real-time target recognition and tracking for autonomously landing an UAV in a cluttered shipboard environment, Autonom. Robot. 41(4)(2017)881-901.

[13] Y. Meng, W. Wang, H. Han, M. Zhang, A visual/inertial integrated landing guidance method for UAV landing on the ship, Aeroap. Sci. Technol. 85(2019)474-480.

[14] Y. Meng, W. Wang, H. Han, M. Zhang, A vision/radar/INS integrated guidance method for shipboard landing, IEEE Trans. Ind. Electron., 66(11)(2019)8803-8810.

[15] H. Hu, Y. Wu, J. Xu, Q. Sun, Path planning for autonomous landing of hlicopter on the aricraft carrier, Mathematics 6(10)(2018)178.

[16] J. Ghommam, M. Saad, Autonomous landing of a quadrotor on a moving platform, IEEE Trans. Aerosp. Electron. Syst. 53(3)(2017)1504-1519.

[17] J. Moon, J. C. Domercant, D. Mavris, A simplified approach to assessment of mission success for helicopter landing on a ship, Int. J. Control Autom. Syst. 13(3)(2015)680-688.

[18] S. M. B. Malaek, N. Sadati, H. Izadi, M. Pakmehr, Intelligent autolanding controller design using neural networks and fuzzy logic, 5th Asian Control Conf. IEEE Publishing House, 1(2004)365-373.

[19] Q. Lu, B. Ren, S. Parameswaran, Shipboard landing control enabled by an uncertainty and disturbance estimator, J. Guid. Control Dyn. 41(7)(2018)1502-1520.

[20] B. Hu, L. Lu, S. Mishra, A control architecture for time-optimal landing of a quadrotor onto a moving platform, Asian J. Control 20(5)(2018)1701-1712.

[21] P. Serra, R. Cunha, T. Hamel, D. Cabecinhas, C. Silvestre, Landing of a quadrotor on a moving target using dynamic image-based visual servo control, IEEE Trans. Robtics 32(6)(2016)1524-1535. 
[22] X. Yang, M. Garratt, H. Pota, Non-linear position control for hover and automatic landing of unmanned aerial vehicles, IET Control Theorey Appl. 6(7)(2012)911-920.

[23] C. K. Tan, J. Wang, Y. C. Paw, F. Liao, Autonomous ship deck landing of a quadrotor using invariant ellipsoid method, IEEE Trans. Aerosp. Electron. Syst. 52(2)(2016)891-903.

[24] C. K. Tan, J. Wang, Y. C. Paw, T. Y. Ng, Tracking of a moving ground target by a quadrotor using a backstepping approach based on full state cascaded dynamics, Appl. Soft Comp. 47(2016)47-62.

[25] C. K. Tan, J. Wang, Y. C. Paw, F. Liao, Robust linear output feedback controller for autonomous landing of a quadrotor on a ship deck, Int. J. Control 92(12)(2019)2791-2805.

[26] Y. Huang, Z. Zheng, L. Sun, M. Zhu, Saturated adaptive sliding mode control for autonomous vessel landing of a quadrotor, IET Control Theorey Appl. 12(13)(2018)1830-1842.

[27] Y. Huang, M. Zhu, Z. Zheng, M. Feroskhanc, Fixed-time autonomous shipboard landing control of a helicopter with external disturbances, Aerosp. Sci. Technol. 84(2019)18-30.

[28] K. Xia, S. Lee, H. Son, Adaptive control for multi-rotor UAVs autonomous ship landing with mission planning, Aerosp. Sci. Technol. 96(2020)105549.

[29] H. Schaub, J. L. Junkins, Analytical Mechanics of Space Systems, Reston:
AIAA, (2003).

[30] T. I. Fossen, Guidance and Control of Ocean Vehicles, John Wiley and Sons Ltd, (1994).

[31] J. A. Farrell, M. Polycarpou, M. Sharma, W. Dong, Command filtered backstepping, IEEE Trans. Autom. Control 54(6)(2009)1391-1395.

[32] L. Wang, J. Su, Trajectory tracking of vertical take-off and landing unmanned aerial vehicles based on disturbance rejection control, IEEE/CAA J. Autom. Sinica 2(1)(2015)65-73.

[33] W. F. Phillips, C. E. Hailey, Review of attitude representations used for aircraft kinematics, J. Aircraft 38(2001)718-737.

[34] A. H. J. De Ruiter, C. J. Damaren, Effect of attitude parameterization on the performance of passivity-based adaptive attitude control, AIAA Guid. Nav. Control Conf. (2001)AIAA-2001-4154.

[35] G. Xing, S. A. Parvez, Nonlinear attitude state tracking control for spacecraft, J. Guid. Control Dyn. 24(3)(2001)624C-626.

[36] Y. Zou, W. Huo, Singularity-free non-linear controller for a model-scaled autonomous helicopter, IET Control Theorey Appl. 10(2)(2016)210-219.

[37] Y. Zou, Nonlinear robust adaptive hierarchical sliding mode control approach for quadrotors, Int. J. Robust Nonlinear Control 27(6)(2016)925941. 